\title{
Managing sudden transportation disruptions under delivery delay and quantity loss
}

\section{Sanjoy Kumar Paul}

UTS Business School, University of Technology Sydney, Australia

\section{Sobhan Asian ${ }^{1}$}

School of Business IT \& Logistics, College of Business, RMIT University, Australia

\section{Mark Goh}

NUS Business School and The Logistics Asia-Pacific

National University of Singapore, Singapore

\section{S. Ali Torabi}

School of Industrial Engineering, College of Engineering, University of Tehran, Tehran, Iran

\begin{abstract}
Transportation disruption, a common source of business interruptions, can cause significant economic loss to a lean supply chain. This paper studies a lean, two-stage suppliermanufacturer coordinated system where a sudden disruption interrupts the transportation network, creating delivery delays, and product quantity losses. We develop a model to generate a recovery plan after a sudden disruption occurrence, to minimize the negative impacts of the disruption. At the same time, given the computational intensity and problem complexity, three heuristics based on the delivery delay and fractional quantity loss caused by a sudden disruption are developed. We conduct a number of numerical experiments to validate our proposed solution methods, and a scenario-based analysis to test the model and analyse the impact of sudden transportation disruption under three disruption scenarios. The performance of presented heuristics against the Generalized Reduced Gradient method are compared. The results reveal that the proposed heuristics can generate a recovery plan accurately and consistently.
\end{abstract}

Keywords: Transportation; Disruption; Supply chain; Recovery plan; Heuristics

\footnotetext{
${ }^{1}$ Corresponding author. Email: sobhan.asian@rmit.edu.au.
} 


\section{Introduction}

In a globally competitive business environment, a well-executed supply chain - which is a network that seamlessly receives inputs or raw materials from suppliers, produces final products at its manufacturing facilities, and delivers them to customers through a distribution network (Paul et al. 2016a) - is crucial to the conduct of business (Min et al. 2008; Wieland and Wallenburg 2013). An efficient supply chain not only promotes the operational performance of the firms, but also contributes significantly to building, sustaining, and winning competitive advantage (Hendricks and Singhal 2003; Asian et al. 2009; Tavakoli et. al. 2012; Somarin et. al. 2016; Somarin et. al. 2017a; Somarin et. al. 2017b).

Today, firms and their attendant supply chains are increasingly being exposed to disruptions. Alcantara and Riglietti (2015) reported that $74 \%$ of the firms from 426 sampled organizations had experienced at least one disruption in their supply chain, with 6 to 20 disruptions a year for $15 \%$ of the responding firms. Their survey also revealed the financial extent of supply chain disruptions, ranging from 50,000 to 500 million Euros. These disruptions not only reduce the operating income but also the return on sales and assets, and increase the production cost and level of inventory (Chowdhury et al. 2016; Y. Kim et al. 2015). Moreover, supply chain disruptions are associated with an abnormal decrease in shareholder value (Hendricks and Singhal 2003). Besides financial loss, supply chain disruptions bring about many other negative outcomes for the firm such as reputation effects $(27 \%)$, reduced productivity (58\%), and lower projected revenue (38\%) (Alcantara and Riglietti 2015). Indeed, the extant literature suggests that firms are prone to significant financial and reputational losses caused by supply chain disruptions (Wagner and SilveiraCamargos 2012). Considering the severe impact of supply chain disruptions, academia and industry have extensively acknowledged supply chain disruption and its management approach (Ansaripoor et al. 2017; Asian et al. 2016; Blome and Schoenherr 2011).

While the awareness of supply chain disruptions has grown, many firms remain exposed to particular types of supply chain disruptions, such as transportation disruption. A transportation disruption refers to a disruption in the transit of goods from one node to another (e.g. supplier to manufacturer) (Hishamuddin et al. 2013). Transportation disruption halts the flow of products between nodes whereas the other types of disruptions stop production (Hishamuddin et al. 2013). Besides, transportation disruption has its specificity, distinctive in that goods in transit are stopped, with all other operations of the supply chain intact (Wilson 2007). While such a disruption delays delivery to a destination, it may also damage the condition of the delivered products especially for the temperature sensitive 
perishable items. Firms across all sectors of industry view transportation disruption to be a major risk, as it delays goods delivery and goods write-offs in their supply chains. The impact can be supply-chain wide (Giunipero and Eltantawy 2004), and cause havoc such as missed delivery deadlines, plant shutdowns, lost sales, and loss of customer goodwill (Guiffrida and Jaber 2008). For instance, in 2010, Kenyan farmers who air-freighted exports to Europe had to destroy over 400 tons of cut-flowers when they faced two days of flight cancellations, due to the eruption of an Icelandic volcano (Chopra and Sodhi 2014). Indeed, the possible disruption cases arising from delivery delays and quantity loss (i.e., only a delivery delay, only a portion of the quantity loss, and both delivery delays and some quantity loss) are practical and pertinent and it can so critical that it demands management attention. Already, industries in Asia are constantly bombarded with this phenomenon in each operating quarter.

The sources of transportation disruptions can include natural disasters, labour disputes, terrorist attacks, and infrastructure failure (Wilson 2007). Transportation disruptions may increase in the near future because of the adverse weather events due to global warming as these events will certainly hamper the operating time window of shipping, air freighting, and rail transport (Chen et al. 2015). A major consequence of a transportation disruption is the delayed delivery to the customer. As a mitigation measure, some researchers have recently developed recovery models that consider delivery delays after a disruption (Hishamuddin et al. 2012, 2014; Xia et al. 2004). Another consequence of a transportation disruption is the loss in product quantity, which has been largely ignored in most previous studies. In this situation, the customer will receive a quantity (ranging from nothing to the almost entire lot) smaller than expected (Wilson 2007). This affects the production scheduling, especially for just-in-time delivery (Chen et al. 2013; Khazaei Pool et al. 2017a).

According to an industry survey conducted by Alcantara and Riglietti (2015), 40\% of the firms are concerned about transportation disruptions, while only about $9 \%$ of them are forming contingency (i.e. business continuity) plans for coping with transportation network disruptions. Admittedly, transportation disruptions are difficult to predict (Chen et al. 2013; Chowdhury et al. 2016; Wagner and Bode 2008; Khazaei Pool et al. 2017b). Hence, formal operational planning is needed to minimize the impact of sudden transportation disruptions. An appropriate recovery model can assist managers to formulate appropriate plans to manage the onset of sudden transportation disruptions. Doing so can help to manage profitability and reduce the losses. However, studies dealing with transportation disruptions especially using recovery plans has received relatively scant attention in the literature (Paul et al. 2016a). In particular, none of the transportation disruption recovery models developed thus far have 
investigated a rolling production planning considering both delivery delay and proportionate quantity loss. Focusing mainly on the economic ordering problem (Choi et al. 2016a; Choi et al. 2016b), the work closest to our study in the literature considers only a delivery delay to develop a recovery "ordering" plan in a different supply chain setting (Hishamuddin et al., 2013). Inspired by this fact, our paper seeks to develop a robust recovery plan for responding to transportation disruptions in a supplier-manufacturer system, considering both consequences of a sudden transportation disruption, namely, delivery delay and partial quantity loss.

The focus of this paper is to develop a model for a two-stage centralized supply chain (consisting of a supplier and a manufacturer) and generate a production recovery plan after the occurrence of a sudden transportation disruption. In particular, the concentration of present work is mainly on mitigating operational impacts/effects (rather than analyzing the transportation disruption as a cause itself) of theafter a sudden transportation disruption on manufacturing planning and scheduling in a two-stage centralized supply chain. Thus, the main objective of this paper is to generate a production recovery plan, which realistically considers both delivery delays and partial quantity losses, after the occurrence of a sudden transportation disruption.

As the computational effort can be complex when solving a large-scale problem instance, therefore, to reduce the computational intensity and complexity involved in finding the optimal solution, three cost efficient heuristics are developed to approximate the optimal recovery plan under different scenarios. Two of the heuristics (partial quantity loss, and both delivery time delay and partial quantity loss) are specially developed for the problems introduced in this paper. Compared to the existing heuristics in the literature, which are mainly developed based on a multiple-step iteration method (Hishamuddin et al. 2013); a greedy approach, which has been successfully implemented in prior studies, is adopted (Paul et al. 2014c, 2015b, 2016a, 2016b). To ensure the accuracy of the proposed recovery policies and enhance the practicality of the developed model, we relax one of the main model assumptions (the fixed number of recovery cycles) and present two new heuristic procedures for determining the best number of recovery cycles in the event of transportation disruption.

Testing our model through an intensive numerical study and conducting a scenariobased analysis, it is shown that the proposed model can effectively help to manage sudden transportation disruptions, and hence enhance a firm's operational performance. To further validate our approach, the heuristics solutions are compared against the standard Generalized Reduced Gradient (GRG) search algorithm. Besides assisting managers to formulate a 
contingency recovery plan to manage transportation disruptions, this solution will equip managers for quick recovery from transportation disruptions, which in turn, will mitigate any disruption impact on a firm's operational activities.

The rest of the study is organized as follows. The literature review and problem description are presented in Sections 2 and 3 respectively. The model formulation and properties are found in Section 4. Section 5 presents the three heuristics. Section 6 contains the algorithm for the random experimentation. Section 7 compares the experimental results with the GRG method and describes the scenario-based analyses. Finally, Section 8 concludes the paper.

\section{Literature review}

Clearly, a smooth and efficient supply chain operation is not assured, unless preparing for supply chain disruptions (Lu et al. 2017). Recently, three review papers published in supply chain risk and disruption management and reported different OR/MS models developed for managing supply chain risk and disruption (Fahimnia et al. 2015; Paul et al. 2016a; Snyder et al. 2016). Independently, Ho et al. (2015) conducted an extensive literature review and summarized the different types of disruptions and risks in the supply chain. However, to date, the topic of transportation disruption has received much less attention compared to the other types of supply chain disruptions.

Unlike the strong assumptions made in the literature (Hishamuddin et al., 2013), transportation disruptions not only delay delivery from one node of a supply chain to another, but they may also damage the actual condition or some portion of the products. Transportation disruption is unique in its nature and can quickly affect an entire supply chain (Giunipero and Eltantawy 2004; Sharifkhani et al. 2016), leading to problems such as late deliveries, operations shutdown, lost sales, and loss of reputation (Guiffrida and Jaber 2008). Considering the severe impact of transportation disruptions, some studies have provided insights to such disruptions. For example, Giunipero and Eltantawy (2004) were the first to discuss transportation disruption albeit they did not offer any risk measurement or mitigation strategies to manage such disruptions. Later, Wilson (2007) investigated the effects on supply chain performance due to transportation disruption between two echelons of a five-echelon supply chain using system dynamics. That study considered four areas of transportation disruption: (i) disruption between the warehouse and the retailer, (ii) disruption between the tier one suppliers and the warehouse, (iii) disruption between the tier two and tier one suppliers, and (iv) disruption between the raw material suppliers and tier two suppliers. 
Similarly, in our study, a two-stage supply chain with a focus on transportation disruption between a tier one supplier and a manufacturer is investigated.

Though there is a lack of model-based research on transportation disruption, several earlier works have been found, which focus on developing models for other types of supply chain disruption recovery planning. Most of these studies have focused on three areas of supply chain disruptions: supply disruption, production disruption, and demand fluctuation (Ho et al. 2015). Recovery models to deal with supply disruptions are by far the most common (Paul et al. 2016a). Weiss and Rosenthal (1992) first developed an optimal inventory policy model for the economic order quantity inventory system, which may face disruptions in either supply or demand. Özekici and Parlar (1999) considered backorders to analyse a production-inventory model under random supply disruptions. Asian and Nie (2014) extended the concept to develop a win-win coordination mechanism for the contract between a buyer and a backup supplier when market demand is uncertain; and the main supplier is subject to supply disruptions. Pal et al. (2012) developed a model for a multiechelon supply chain, comprising two suppliers (a main and a backup supplier) delivering raw materials to a manufacturer, whereby the main supplier may face a random supply disruption and the secondary supplier is perfectly reliable but more expensive than the main supplier. Hishamuddin et al. (2013) also developed a recovery model to manage transportation disruption in a two-echelon supply chain. However, similar to the other aforementioned papers, that study focused on a supplier-retailer system to develop a recovery model for "ordering" decisions by considering only delivery delays. In our paper, to develop a production-planning recovery model, both delivery time delay and partial quantity losses due to transportation disruptions are taken into account. Another aspect, which distinguishes our paper from the rest especially that of Hishamuddin et al. (2013), is our proposed greedy heuristics solution approach, which is different from the existing iterative based methods, and generates better and reliable results.

On recent supply disruption recovery models, Hishamuddin et al. (2014) applied the backorders and lost sales concept for managing supply disruptions in a two-stage singlesupplier, single-retailer supply chain. The study was extended by Paul et al. (2014b) and (Paul et al. 2016b), who developed a recovery model for managing supply disruptions in a three-stage supply chain. They considered backorders, lost sales, and outsourcing options to generate optimal recovery plans. Other recent works related to developing supply disruption mitigation strategies can be found in Yang et al. (2009), Li et al. (2010), Shao and Dong (2012), Chung et al. (2015), Guo et al. (2013), Zhang et al. (2013), Hu et al. (2013), Yan et 
al. (2014), Pal et al. (2014), Ray and Jenamani (2016), Do et al. (2016), Faghih-Roohi et al. (2015), Mogre and D'Amico (2016), and Li et al. (2015). Readers interested in supply disruption management are referred to Snyder et al. (2016) and Paul et al. (2016a, 2017).

As mentioned, in developing the recovery models, several studies have focused on production disruption, also known as process disruption. For instance, Xia et al. (2004) developed a general disruption management approach for a two-stage production and inventory control system that incorporated a penalty cost for the deviations of the revised plan from the original. They divided the disruption interval as follows: pre-disruption, indisruption, and post-disruption, to eke out a detailed analysis of the disruption effects. Later, Hishamuddin et al. (2012) extended the study to develop a recovery plan from a single production disruption for a single stage production-inventory system. This was further extended to a real-time disruption recovery plan from both single and multiple production disruptions for single-stage (Paul et al. 2013, 2015b), two-stage (Paul et al. 2014c), and threestage (Paul et al. 2015a) production-inventory systems. They incorporated backorders, lost sales and/or outsourcing options to generate the recovery plan and developed some solution heuristics. Other studies (Baghalian et al. 2013; Kim 2013; Tang et al. 2012) have considered demand fluctuations when formulating a supply chain recovery model. Paul et al. (2014c) have applied a real-time recovery concept on a supplier-retailer coordinated system for managing demand fluctuations under backorders and lost sales.

Though delivery delays and quantity losses are the most common consequences of a sudden transportation disruption, our survey highlights that no study has so far developed a recovery model for production planning where both delivery delays and fractional quantity loss simultaneously occur (Hishamuddin et al. 2012; Wilson 2007). To fill this research gap, we focus on a two-stage supplier-manufacturer supply chain and develop a model to generate a production recovery plan after the occurrence of a sudden transportation disruption. Both delivery delays and fraction of quantity losses resulting from a sudden transportation disruption are considered, and both backorders and lost sales are applied. Further, given the considerable cost of commercial software and in order to reduce the computational intensity and complexity involved in finding the optimal solution, we develop three heuristics to approximate the optimal recovery plan. The recovery plan under all possible disruption scenarios arising from delivery delays and quantity loss (i.e. only a delivery delay, only a portion of the quantity loss, and both delivery delay and some quantity loss) are generated.

From what we know, this is the first model, which develops a recovery model in a two-stage supplier-manufacturer coordinated system considering both delivery delays and 
fractional quantity loss due to a sudden transportation disruption. We apply the heuristics on a number of test problems, which are generated randomly using a uniform distribution. The heuristics solutions are compared with those obtained by the GRG method for 100 random test problems. Further, a random experimentation is designed to analyse the effect of randomly generated disruption events that are not known a priori. A detailed sensitivity analysis is performed to explain the usefulness of the developed model. After comparing and validating our proposed heuristics, we analyse the results for different types of disruption scenarios. To this aim, the scenarios from all possible consequences led by sudden transportation disruptions are randomly generated and categorised as low, medium, and high. The main contributions of this paper can be summarized as follows:

i. Develop a new mathematical formulation to manage sudden transportation disruptions, which cannot be predicted in advance. The model considers both delivery delays and partial quantity loss as the consequences of a sudden transportation disruption.

ii. Propose three heuristics for generating recovery plans for a sudden transportation disruption. The recovery plans are generated after the occurrence of a disruption covering a finite number of upcoming periods on a real-time basis to minimize the total loss due to disruptions.

\section{Problem Description}

Our research seeks to formulate a mathematical model for an efficient recovery plan under a sudden transportation disruption where both delivery delays and quantity losses exist. In this research, a two-stage supplier-manufacturer coordinated supply chain system is considered. Under normal conditions, such this system can be coordinated by continuous batch production (Sarker and Khan 1999) and a joint Economic Production Quantity (EPQ) model

(Banerjee 1986). Figure 1 presents an ideal system where no transportation disruption occurs and, hence, the optimal batch quantities $\left(Q^{*}\right.$ and $\left.q^{*}\right)$ are found from the EPQ model. When the supplier completes a batch production of $Q$, the batch is delivered to the manufacturer through the transport network. We consider the supplier's set-up time $\left(S_{t s}\right)$ and idle time $\left(T_{s}\right)$ between two consecutive production batches. Once the manufacturer receives a batch, he starts to make the final product to satisfy downstream customer annual demand $(D)$. Let $q$ denote the manufacturer's lot size, which is assumed to be completely delivered to the customer. A coordinated (supplier-manufacturer) system is considered, where $\mathrm{N}=$ number of units of raw materials required for producing one unit final product - can be obtained from 
Bill of Materials (BOM). Hence, the batch production of $Q$ can be expressed as $Q=N q$.

The manufacturer has set-up time $\left(S_{t m}\right)$ and idle time $\left(T_{m}\right)$ between two consecutive batches.

In such a lean production environment, a sudden transportation disruption can interrupt the fragile flow of raw materials and cause delayed delivery and/or quantity loss. The main target is then to revise the current inventory and production plans after the occurrence of a transportation disruption, known as the recovery plan, so that the negative impacts can be minimized.

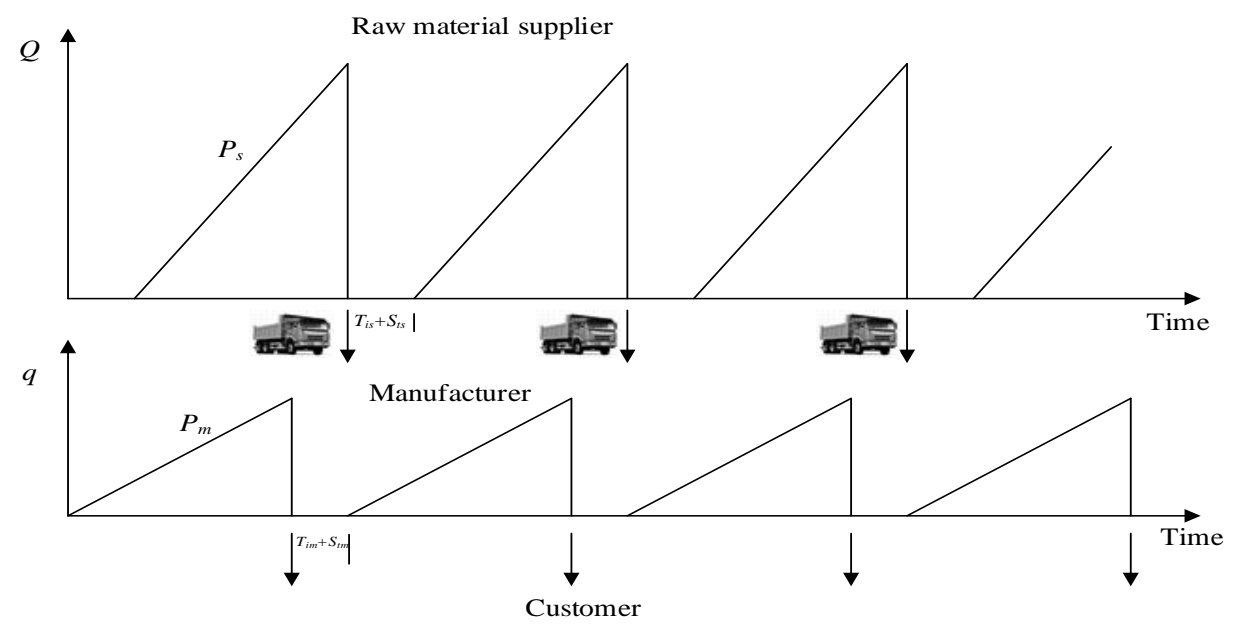

Fig. 1: Ideal two-stage supply chain

In Figure 2, the dotted line represents the recovery plan after a sudden transportation disruption which mimics a delayed delivery of time $\left(T_{d}\right)$ and a fraction of quantity loss $(f)$. After the occurrence of a transportation disruption, the production-inventory plans are revised for a finite number of upcoming periods, which is known as the recovery time window (Paul et al. 2014c). Within this recovery time window, the plan will revise both the lot sizes of the supplier and manufacturer ( $X_{i}$ and $Y_{i}$ respectively) to minimize the effects of the transportation disruption.

In practice, the most common consequence of a disruption is delivery delays to a customer. To overcome this consequence, a few researchers developed recovery models considering delivery delays after a disruption (Hishamuddin et al. 2012, 2014; Xia et al. 2004). Another significant consequence of a transportation disruption is quantity loss, which can range from $0 \%$ to $100 \%$ of the lot (Wilson 2007). In this case, the customer will receive less than expected and as a result, the production plan will be distorted. Hence, it is important to simultaneously consider both consequences (delivery delays and quantity loss) while developing a recovery plan after a sudden transportation disruption. To make the model realistic, both delivery delay and quantity loss are considered: 
i. Delivery delay: The raw materials are delivered to the manufacturer with a delay $\left(T_{d}\right)$, which is equal to the disruption duration.

ii. Quantity loss: There is a fraction $(f)$ of quantity loss due to the disruption, set as $(0,1)$ of the delivery lot. The fraction of loss could be anything between 0 and 1 , hence, 0 means no loss and 1 means $100 \%$ loss.

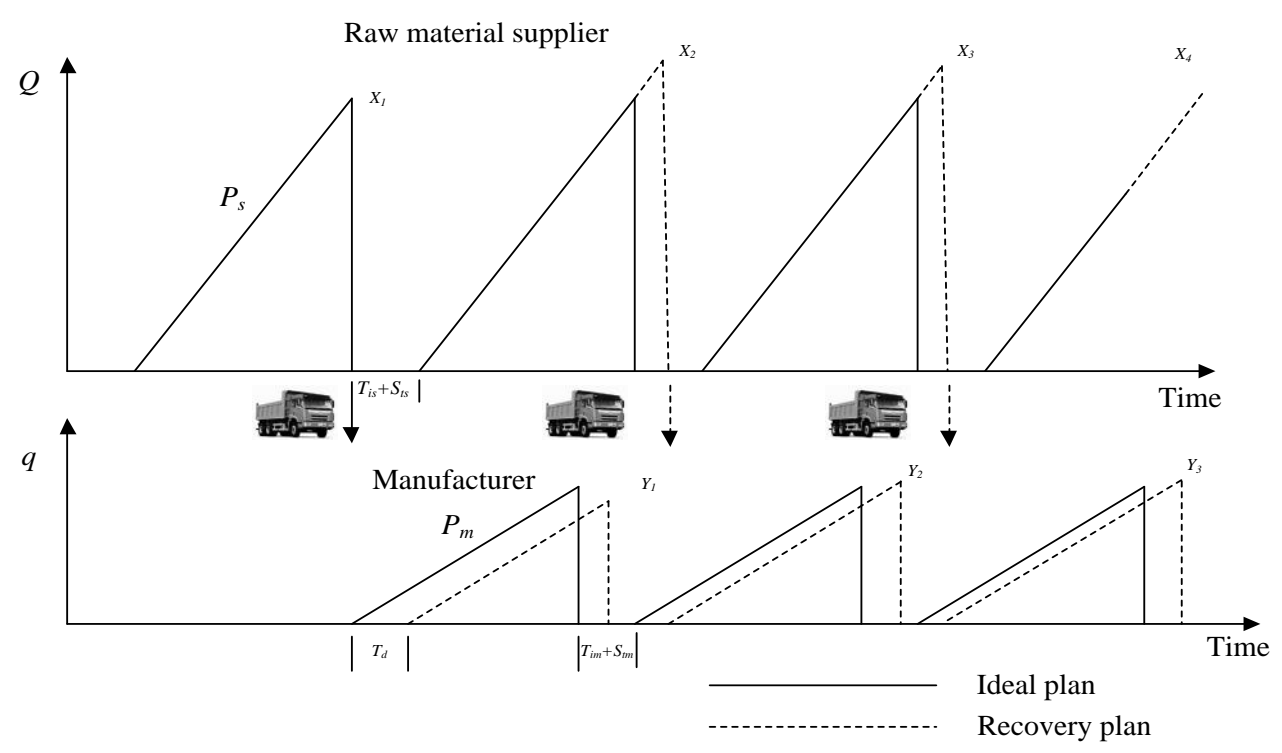

Fig. 2: Recovery plan after the occurrence of a transportation disruption

While both factors are direct consequences of a sudden transportation disruption, the extent to which each of them affects the trade-off between backorders and lost sales costs is different. In other words, given the several existing interdependencies among the model parameters and depending on the consequence of the transportation disruption (i.e., delivery delay and/or quantity loss), supply chain actors behave differently towards the recovery planning problem. Inspired by above facts, the purpose of this study is to develop the recovery planning solutions (revising the production and inventory planning) that can be implemented to hedge against the backorders and lost sales risks under sudden transportation disruptions. To characterize the complexity of the described decision making problem, three scenarios are considered that cover all possible cases led by a sudden transportation disruption: (i) only a delivery delay $\left(T_{d}\right)$, (ii) only a fraction of quantity loss $(f)$, and (iii) both a delivery delay and a partial quantity loss (both $T_{d}$ and $f$ ).

Future supply chain plans are revised after an occurrence of a disruption for the three scenarios. Our main objective in all the scenarios is to minimize the total cost within the recovery time window subject to the production, supply, demand, delivery, and time constraints. 


\section{Model formulation}

We now formulate the mathematical models for both the ideal and recovery plans. In the ideal plan, a joint EPQ model and the optimal batch sizes are determined by minimizing the total holding and set-up costs. For the recovery plan, however, additional backorders and lost sales costs are considered as recovery policies within the recovery time window. To provide a better demonstration, the major associated costs of sudden transportation disruptions are as follows.

i. Backorders: If the system is disrupted, the backorders represent the portion of demand that cannot be fulfilled at the scheduled time but will be delivered at a later date when they are available (Paul et al. 2014c). This policy utilizes the idle times within the recovery time window to satisfy the unfulfilled demand.

ii. Lost sales: When the system is incapable of filling demand after a disruption and customers do not wait for stock to be replenished, demand is lost (Paul et al. 2014c). The following notations are used in our paper for the mathematical models.

Q Supplier lot size

$q$ Manufacturer lot size

$D$ Annual demand (Quantity/year)

$N$ Number of raw material units required to produce one unit of final product

$P_{m}$ Production rate of manufacturer (Quantity/year), $P_{m}>D$

$P_{S}$ Production rate of supplier (Quantity/year), $P_{S}>N D$

$H_{1}$ Holding cost per unit per year for supplier (\$/unit/year)

$\mathrm{H}_{2}$ Holding cost per unit per year for manufacturer (\$/unit/year)

$S_{1}$ Set-up cost for supplier (\$/set-up)

$S_{2}$ Set-up cost for manufacturer (including a fixed pre-determined transportation cost from supplier to manufacturer) (\$/set-up)

$S_{t s}$ Set-up time of supplier (year)

$S_{t m}$ Set-up time of manufacturer (year)

$T_{S}$ Idle time between two consecutive lots for supplier (year)

$T_{m}$ Idle time between two consecutive lots for manufacturer (year)

$T_{d}$ Delivery delay (year)

$f \quad$ Fraction of quantity loss

$U_{d}$ Quantity lost $=f q$

$X_{i}$ Revised lot size of supplier in cycle $i$ in the recovery plan 
$Y_{i} \quad$ Revised lot size of manufacturer in cycle $i$ in the recovery plan

$B_{q i}$ Backorder quantity in cycle $i$ in the recovery plan

$d_{i m}$ Delay in delivery in cycle $i$ to customer in the recovery plan

$n$ Number of cycles in the recovery time window

\section{$n \_\max$ Maximum number of cycles in the recovery window}

$B$ Unit backorder cost (\$ per unit per year)

$L \quad$ Unit lost sales cost (\$ per unit)

To make the models tractable and to obtain insightful results, the following assumptions are made:

i. A single item is produced in the system.

ii. The productions rates $\left(P_{m}\right.$ and $\left.P_{s}\right)$ are greater than the demand rate.

iii. There is no inventory buffer and safety stock in the system, i.e. a truly lean system.

iv. The recovery plan begins after the occurrence of a disruption in transportation.

v. No emergency sourcing is possible.

vi. Partial delivery is acceptable.

To develop and analyse the recovery plans in a supply chain, without losing generality; it is assumed that a single item is produced in the system (Hishamuddin et al. 2012, 2013; Xia et al. 2004). Following previous studies (Hishamuddin et al. 2012) it is assumed that the production rates exceed the demand rate. We also assume that there is no inventory buffer / safety stock as their cost is too expensive. In the recovery plan, it is considered that sudden disruptions can happen in a transportation network and to make the disruption recovery meaningful in practice, the recovery plan will be generated just after a transportation disruption is experienced. In short, the recovery plan is reactive and generated in real-time. We consider both backorder and lost sale policies to recover from a sudden transportation disruption, which are also widely used in both the literature and in practice (Hishamuddin et al. 2012; Paul et al. 2014c). It is assumed that emergency outsourcing is not possible as it is expensive and partial delivery is acceptable to make the recovery plan optimal. Finally, it is assumed that the manufacturer decides on the number of periods in the recovery time window. We later relax this assumption and present two heuristic procedures for determining the best number of recovery cycles in the event of transportation disruption.

\subsection{Ideal plan model}


To better present the research problem, we first focus on an ideal situation where no disruption affects the transportation network. In the ideal state, a continuous batch production system (Sarker and Khan 1999) is considered, and the optimal lot size is determined by using the EPQ model (Banerjee 1986). Following the literature, holding and set-up costs for the supplier and manufacturer are considered. The associated cost equations are presented in Eqs. (1) - (5). We then determine the optimal lot sizes for the manufacturer and supplier by Eqs. (6) and (7), respectively. Further, the idle time between two consecutive batches for both the supplier and the manufacturer are defined by Eqs. (8) and (9).

Supplier holding cost $=\frac{Q}{2} H_{1} \frac{N D}{P_{S}}$

Supplier set-up cost $=\frac{N D}{Q} S_{1}$

Manufacturer holding cost $=\frac{q}{2} H_{2} \frac{D}{P_{m}}$

Manufacturer set-up cost $=\frac{D}{q} S_{2}$

Total cost $(T C)=\frac{Q}{2} H_{1} \frac{N D}{P_{S}}+\frac{N D}{Q} S_{1}+\frac{q}{2} H_{2} \frac{D}{P_{m}}+\frac{D}{q} S_{2}$.

With $Q=N q$, the total cost function can be derived as follows:

Total cost $(T C)=\frac{N q}{2} H_{1} \frac{N D}{P_{S}}+\frac{D}{q} S_{1}+\frac{q}{2} H_{2} \frac{D}{P_{m}}+\frac{D}{q} S_{2}$

Under a centralized system and to achieve economies of scale in distribution of raw material and finished products' expenses, we assume that the manufacturer is responsible for transportation and delivery operations. Hence, for each manufacturer's production run, a fixed major set-up cost $\left(\mathrm{S}_{2}\right)$ is considered, which includes some minor set-up cost components as well as a pre-determined fixed transportation cost per shipment from the supplier to manufacturer, regardless of quantity of items ordered. This is a reasonable assumption and consistent with existing relevant studies in literature (Chen and Chen, 2005; Daskin et al., 2005).

To minimize the total cost, we set $\frac{d}{d q}(T C)=0$.

After taking derivatives and some simplifications, the optimal manufacturer lot size is derived as Eq. (6).

$q=\sqrt{\frac{2\left(S_{1}+S_{2}\right)}{\frac{N^{2}}{P_{S}} H_{1}+\frac{H_{2}}{P_{m}}}}$.

Optimal supplier lot size, $Q^{*}=N q$

Idle time between two consecutive lots for supplier, $T_{s}=\frac{Q}{N D}-\frac{Q}{P_{S}}-S_{t s}$ 
Idle time between two consecutive lots for manufacturer, $T_{m}=\frac{q}{D}-\frac{q}{P_{m}}-S_{t m}$

\subsection{Mathematical model for recovery plan}

We develop a mathematical model to generate the recovery plan after the occurrence of a sudden disruption in transportation. Similar to the ideal plan, the key equations for the different costs are first determined and the model is formulated as a constrained mathematical programming problem. To better capture the transportation disruption economic impacts, two additional costs are considered, namely, backorders and lost sales, under the recovery plan. The backorders cost is defined as the unit backorder cost multiplied by the backorder units and the associated time delay (Paul et al. 2015a). The lost sales, however, is determined as the unit lost sales cost multiplied by the lost sales units (Paul et al. 2015a). To be consistent, the structure of "set-up" and "holding" costs are considered to be the same as the ideal scenario, where the transportation cost is incorporated in the manufacturer's set-up costs as one of the fixed cost components. However, in contrast to the ideal plan, the focus here is on minimizing the total cost of the supplier and the manufacturer during the recovery plan window.

The different costs under the recovery plan are calculated as follows:

Set-up cost at supplier $=S_{1} n$

Holding cost at supplier $=\frac{1}{2} H_{1} X_{1} \frac{X_{1}}{P_{S}}+\frac{1}{2} H_{1} X_{2} \frac{X_{2}}{P_{S}}+\cdots+\frac{1}{2} H_{1} X_{n} \frac{X_{n}}{P_{S}}$

$$
=\frac{H_{1}}{2 P_{S}}\left[\sum_{i=1}^{n} X_{i}^{2}\right]
$$

Set-up cost at manufacturer $=S_{2} n$

Holding cost at manufacturer $=\frac{1}{2} H_{2} Y_{1} \frac{Y_{1}}{P_{m}}+\frac{1}{2} H_{2} Y_{2} \frac{Y_{2}}{P_{m}}+\cdots+\frac{1}{2} H_{2} Y_{n} \frac{Y_{n}}{P_{m}}$

$$
=\frac{H_{2}}{2 P_{m}}\left[\sum_{i=1}^{n} Y_{i}^{2}\right]
$$

Lost sales cost $=L$ (units produced in ideal plan - units produced in revised plan)

$$
=L\left[n q-\sum_{i=1}^{n} Y_{i}\right]
$$

Backorder cost $=B *$ Backorder units $*$ delay

$$
=B \sum_{i=1}^{n} Y_{i}\left[d_{i m}\right]
$$

where

$d_{1 m}=T_{d}+\frac{Y_{1}}{P_{m}}-\frac{q}{P_{m}}$ 


$$
d_{i m}=\max \left(\frac{q}{D}, T_{d}+\frac{Y_{1}}{P_{m}}\right)+\sum_{j=2}^{i} \frac{Y_{j}}{P_{m}}+(j-1) s_{t m}-\frac{(j-1) q}{D}-\frac{q}{P_{m}} ; \forall i \neq 1
$$

The objective function (total cost function) is derived in Eq. (16) by adding the all the cost presented in Eqs. (10) - (15). In such a problem setting, the manager attempts to actively revise the production plan and effectively satisfy the customers' demand. A critical question then arises after the occurrence of a transportation disruption is: how to minimize the expected cost function, which is now subject to the supply, production, demand, and delivery constraints, during the recovery time window. These constraints are presented by Eqs. (17) (24).

$$
\operatorname{Min}\left(X_{i}, Y_{i}\right)=S_{1} n+\frac{H_{1}}{2 P_{s}}\left[\sum_{i=1}^{n} X_{i}^{2}\right]+S_{2} n+\frac{H_{2}}{2 P_{m}}\left[\sum_{i=1}^{n} Y_{i}^{2}\right]+L\left[n q-\sum_{i=1}^{n} Y_{i}\right]+B \sum_{i=1}^{n} Y_{i} d_{i m}
$$

subject to constraints (17) $-(22)$,

$$
\begin{aligned}
& X_{1}=Q \\
& \sum_{i=1}^{n} X_{i} \leq n Q+U_{d} N \\
& \sum_{i=1}^{n} Y_{i} \leq n q \\
& X_{i} \leq Q+P_{s} \times \min \left(T_{s}, T_{m}\right) ; \forall i \neq 1 \\
& Y_{i} \leq q+P_{m} \times \min \left(T_{s}, T_{m}\right) ; \forall i \neq 1 \\
& X_{i}, Y_{i} \geq 0 ; \forall i .
\end{aligned}
$$

To make mathematical models tractable, focus on the main aspects of our research objective, and obtain insightful results, we assume that the system has enough truck capacity to deliver the raw materials from the supplier to manufacturer. This assumption can be justified by knowing the fact that, in our model setting, the disruption consequence is actually quantified in terms of the transported batch size and, hence, the truck capacity has no direct impact on the decision making process.

Constraint (17) presents the supplier's lot size constraint for the first cycle. Constraints (18) and (19) present the total production for the supplier and manufacturer respectively. Constraints (20) and (21) present the supplier's and manufacturer's lot sizes for each cycle in the recovery window. Finally, Constraint (22) presents the non-negativity constraints.

With transportation disruption, the centralized planner aims to minimize the risks of backorders and lost sales via the recovery plan. Specifically, the manufacturer and supplier need to consider their constraints and make their best decisions at post-disruption on the following. What are the optimal lot sizes in a recovery time window to minimize the impact 
of the disruption? Taking the above decision, the supply chain members establish a practical recovery plan, which enables them to effectively respond to the market demand and minimize the overall expected cost of the system.

As mentioned, to eliminate the cost of a commercial solver software and reduce the computational intensity and complexity involved in finding the optimal solution, we develop a heuristic approach, which can accurately approximate the optimal recovery plan. To fulfil this intention, the focus is put on finding the model properties by developing a few properties, which enable us to develop the heuristics and design the experimentation.

\subsection{Model properties}

So far, our focus has been on describing the system and formulating the problem using a constrained mathematical programming model. Now, we turn our attention to the recovery policy under each of the three possible disruption scenarios, described in Section 3. The special conditions of each disruption scenario are considered to characterize some of the main properties of the optimal solution. This will help us to better understand the trade-offs between the backorders and lost sales costs made under different situations.

Property 1: Lost sales will be more attractive in the optimal solution if $B \times T_{d}>L$ and backorders will be more attractive if $B \times T_{d} \leq L$.

Proof: After a disruption, the delay time is $T_{d}$ within the recovery time window. In this situation, the unit backorder cost is $B \times T_{d}$, whereas the unit lost sales cost is $L$. In order to develop an effective recovery plan, one must trade-off between the backorders and lost sales costs. So, if $B \times T_{d}>L$, lost sales will be more attractive than the backorders in the optimal solution and if $B \times T_{d} \leq L$, then backorders will be more attractive.

\section{(a) For both delivery delay $\left(T_{d}\right)$ and a partial quantity loss $(f)$}

Property 2: When backorders are more attractive, the recovery plan will incorporate only backorders if $T_{d}+\frac{U_{d}}{P_{m}} \leq n \times \min \left(T_{s}, T_{m}\right)$ and both backorders and lost sales if $T_{d}+\frac{U_{d}}{P_{m}}>$ $n \times \min \left(T_{s}, T_{m}\right)$.

Proof: After a sudden transportation disruption has occurred, we have: Unfulfilled demand= $U_{d}$ and disruption duration $=T_{d}$. Therefore, the utilizable idle time in a cycle is equivalent to $\min \left(T_{s}, T_{m}\right)$.

Knowing that there are $n$ cycles in the revised plan, the total utilisable idle time in the revised planning window is $n \times \min \left(T_{s}, T_{m}\right)$. Moreover, the minimum time needed to recover is 
$T_{d}+\frac{U_{d}}{P_{m}}$. As a result, if $T_{d}+\frac{U_{d}}{P_{m}} \leq n \times \min \left(T_{s}, T_{m}\right)$, the production process is capable to recover the total loss time by using only the idle timeslots. Hence, the recovery plan will incorporate only backorders. If $T_{d}+\frac{U_{d}}{P_{m}}>n \times \min \left(T_{s}, T_{m}\right)$, the production process is incapable of recovering by using the idle timeslots. Hence, it will incorporate lost sales as well, so the recovery plan will incorporate both backorders and lost sales.

\section{(b) For only delivery delay $\left(T_{d}\right)$}

Property 3: When backorders are more attractive, the recovery plan will incorporate only backorders if $T_{d} \leq n \times \min \left(T_{s}, T_{m}\right)$ and both backorders and lost sales if $T_{d}>n \times$ $\min \left(T_{s}, T_{m}\right)$.

\section{Proof: Same as Property2.}

\section{(c) For only a partial quantity loss $(f)$}

Property 4: When backorders are more attractive, the recovery plan will incorporate only

backorders if $\frac{U_{d}}{P_{m}} \leq n \times \min \left(T_{s}, T_{m}\right)$, and both backorders and lost sales if $\frac{U_{d}}{P_{m}}>n \times$ $\min \left(T_{s}, T_{m}\right)$.

\section{Proof: Same as Property 2.}

The above properties will enable us to simplify the solution process as we present some heuristics to approximate an optimal decision.

\section{Solution approaches}

In this Section, we develop three heuristics for solving the disruption problems and two approaches for determining $n$ if it is unknown.

\subsection{Proposed Heuristics}

We now consider the important properties obtained from the above properties and develop three heuristics for managing sudden transportation disruptions in the supply chain. Focusing on the particular consequences of a sudden disruption, the heuristics are developed separately for different scenarios as follows:

\section{i. Heuristic 1: when the disruption consequence is only a delivery delay $\boldsymbol{T}_{d}$}

First, a heuristic for managing transportation disruption with the consequence of only a delivery delay denoted by $T_{d}$ is developed. In Step 1 , the variables in the ideal plan are determined. The delivery delay time is given as the input in Step 2. If $B \times T_{d}>L$, the recovery plan is determined from Step 3, which involves only lost sales, as presented in Property 1 . If $B \times T_{d} \leq L$, the recovery plan is determined using Step 4, which involves both 
backorders and lost sales, as shown in Property 3. Step 5 determines the different costs as well as the backorders and lost sales quantities. The results are recorded in Step 6. Finally, Step 7 terminates the program. The above steps are described as follows.

Step 1: Determine the variables of the ideal plan using Eqs. (6) - (9).

Step 2: Input disruption duration $\left(T_{d}\right)$ in the heuristic.

Step 3: If $B \times T_{d}>L$, then we would have

$$
\begin{gathered}
X_{1}=Q \\
X_{2}=Q-T_{d} \times P_{m} \times N \\
X_{i}=Q ; \forall i \neq 1,2 \\
Y_{1}=\left(\frac{q}{P_{m}}-T_{d}\right) \times P_{m} \\
Y_{i}=q ; \forall i \neq 1 .
\end{gathered}
$$

Step 4: If $B \times T_{d} \leq L$, then we would have

$$
\begin{aligned}
& \text { If } T_{d} \leq n \times \min \left(T_{s}, T_{m}\right), \\
& \qquad \begin{array}{c}
X_{i}=Q ; \forall i \\
Y_{i}=q ; \forall i .
\end{array} \\
& \text { If } T_{d}>n \times \min \left(T_{s}, T_{m}\right), \\
& \qquad \begin{array}{c}
X_{i}=Y_{i} \times N ; \forall i \\
Y_{1}=q
\end{array} \\
& Y_{2}=q-\left[T_{d}-n \times \min \left(T_{s}, T_{m}\right)\right] \times P_{m} \\
& Y_{i}=q ; \forall i \neq 1,2 .
\end{aligned}
$$

Step 5: Determine the different costs as well as the backorders and lost sales quantities.

Step 6: Record results.

Step 7: Stop.

\section{ii. Heuristic 2: when the disruption consequence is a partial quantity loss $(f)$}

The steps of Heuristic 2 where the disruption only affects the delivery quantity are described as follows:

Step 1: Determine the variables of the ideal plan using Eqs. (6)-(9).

Step 2: Input of the partial damaged quantity $(f)$ in the heuristic.

Step 3: If $B \times \frac{U_{d}}{P_{m}}>L$, then we would have

$$
\begin{gathered}
X_{i}=Q ; \forall i \\
Y_{1}=(1-f) q
\end{gathered}
$$




$$
Y_{i}=q ; \forall i \neq 1
$$

Step 4: If $B \times \frac{U_{d}}{P_{m}} \leq L$, then we would have

$$
\begin{gathered}
\text { If } \frac{U_{d}}{P_{m}} \leq n \times \min \left(T_{s}, T_{m}\right), \\
X_{1}=Q \\
X_{i}=Y_{i} \times N ; \forall i \neq 1 \\
Y_{1}=(1-f) q \\
Y_{i}=q+\frac{U_{d}}{n-1} ; \forall i \neq 1,2 . \\
\text { If } \frac{U_{d}}{P_{m}}>n \times \min \left(T_{s}, T_{m}\right), \\
X_{i}=Y_{i} \times N ; \forall i \neq 1 \\
X_{1}=(1-f) q, Y_{i}=q+\min \left(T_{s}, T_{m}\right) \times P_{m} ; \forall i \neq 1 .
\end{gathered}
$$

Step 5: Determine the different costs as well as the backorders and lost sales quantity.

Step 6: Record results.

Step 7: Stop.

\section{iii. Heuristic 3: when the disruption consequence is both a delivery delay $\left(T_{d}\right)$ and} a partial quantity loss $(f)$.

The steps of Heuristic 3 where both a delivery delay and partial quantity loss exist are as follows:

Step 1: Determine the variables of the ideal plan using Eqs. (6)-(9).

Step 2: Input disruption duration $\left(T_{d}\right)$ and fraction of damaged quantity $(f)$ in the heuristic.

Step 3: If $B \times T_{d}>L$, then we would have

$$
\begin{gathered}
X_{i}=Q ; \forall i \\
Y_{1}=\min \left[\left(\frac{q}{P_{m}}-T_{d}\right) \times P_{m},(1-f) q\right], Y_{i}=q ; \forall i \neq 1 .
\end{gathered}
$$

Step 4: If $B \times T_{d} \leq L$, then we would have

$$
\begin{aligned}
& \text { If } T_{d}+\frac{U_{d}}{P_{m}} \leq \min \left(T_{s}, T_{m}\right), \\
& \qquad \begin{array}{c}
X_{1}=Q \\
X_{i}=Y_{i} \times N ; \forall i \neq 1
\end{array} \\
& Y_{1}=(1-f) q, Y_{2}=q+U_{d}, Y_{i}=q ; \forall i \neq 1,2 . \\
& \text { If } \min \left(T_{s}, T_{m}\right)<T_{d}+\frac{U_{d}}{P_{m}} \leq 2 \times \min \left(T_{s}, T_{m}\right), \\
& X_{1}=Q
\end{aligned}
$$




$$
\begin{gathered}
X_{i}=Y_{i} \times N ; \forall i \neq 1 \\
Y_{1}=(1-f) q, Y_{2}=q+\frac{U_{d}}{2}, Y_{3}=q+\frac{U_{d}}{2}, Y_{i}=q ; \forall i \neq 1,2,3 . \\
\text { If }(n-1) \times \min \left(T_{s}, T_{m}\right)<T_{d}+\frac{U_{d}}{P_{m}} \leq n \times \min \left(T_{s}, T_{m}\right), \text { then } \\
X_{1}=Q \\
X_{i}=Y_{i} \times N ; \forall i \neq 1 \\
Y_{1}=(1-f) q, Y_{i}=q+\frac{U_{d}}{n-1} ; \forall i \neq 1 . \\
\text { If } T_{d}+\frac{U_{d}}{P_{m}}>n \times \min \left(T_{s}, T_{m}\right), \\
\text { If } T_{d} \leq n \times \min \left(T_{s}, T_{m}\right), \text { then } \\
X_{1}=Q \\
X_{i}=Y_{i} \times N ; \forall i \neq 1 \\
Y_{1}=(1-f) q \\
Y_{i}=q+\left(\frac{n \times \min \left(T_{s}, T_{m}\right)-T_{d}}{n-1}\right) \times P_{m} ; \forall i \neq 1 . \\
\text { If } T_{d}>n \times \min \left(T_{s}, T_{m}\right), \text { then } \\
X_{1}=Q \\
X_{i}=Y_{i} \times N ; \forall i \neq 1 \\
(1-f) q, Y_{i}=q-\left(\frac{\min \left(\frac{U_{d}}{P_{m}}, T-n \times \min \left(T_{s}, T_{m}\right)\right)}{n-1}\right) \times P_{m} ; \forall i \neq 1 .
\end{gathered} .
$$

Step 5: Determine the different costs, the backorders, and lost sales quantity.

Step 6: Record the results.

Step 7: Stop.

\subsection{Determination of $\boldsymbol{n}$ if it is unknown}

In this Section, we propose a heuristic procedure for determining the best number of recovery cycles in the event of transportation disruption. The procedure is as follows.

$i$. Determination of $n$ for a delivery delay

The steps for determining number of recovery cycles for a delivery delay are presented as follows.

\section{Step 1: if $B \times T_{d} \leq L$}

Determine $n_{-} \max =\left\lceil\frac{T_{d}}{\min \left(T_{m}, T_{m}\right)}\right]$ 
Step 2: for $n=1$ to $n \_$max

Run the approach developed in heuristic 1 and calculate backorder and lost sales cost using (14) and (15) for each $n$.

Step 3: choose $n$ for minimum total cost

Step 4: if $B \times T_{d}>L$

$n \_\max =0$

Backorder cost $=0$

Lost sales cost $=L \times T_{d} \times P_{m}$

Step 5: stop

ii. Determination of $n$ for a partial quantity loss

The steps for determining number of recovery cycles for a partial quantity loss are presented as follows.

Step 1: if $B \times \frac{U_{d}}{P_{m}} \leq L$

Determine $n \_\max =\left\lceil 1+\frac{U_{d}}{\min \left(\frac{P_{s} \times T_{s}}{N}, P_{m} \times T_{m}\right)}\right\rceil$

Step 2: for $n=n+1$ to $n \_\max$

Run the approach developed in heuristic 2 and calculate backorder and lost sales cost using

(14) and (15) for each $n$.

Step 3: choose $n$ for minimum total backorder and lost sales cost

Step 4: if $B \times \frac{U_{d}}{P_{m}}>L$

$n \_\max =0$

Backorder cost $=0$

Lost sales cost $=L \times f q$

Step 5: stop 


\section{Random experimentation}

For a fair comparison between our proposed approach and those found in the extant literature, the prior similar studies (Paul et al. 2014c, 2015b, 2016b) are used as benchmarks in order to choose the justifiable ranges and values for the model parameters. Hence, the following range of disruption parameters are applied for the random experimentation: $T_{d}=[0.0001,0.08]$; $f=(0,1)$. The random experiments are then constructed by using the following steps:

Step 1: Input all data for the ideal plan.

Step 2: Determine $q, Q, T_{s}$ and $T_{m}$ for the ideal plan.

Step 3: Generate a random disruption situation

a. For only a delivery delay $T_{d}$

Generate a random number for $T_{d}$

Run Heuristic 1

b. For both a delivery delay $T_{d}$ and a fraction of quantity loss $f$

Generate random number for $T_{d}$ and $f$

Run Heuristic 2

c. For only a fraction of quantity loss $f$

Generate a random number for $f$

Run Heuristic 3

Step 4: Repeat Step 3 1,000 times for each disruption situation.

Step 5: Record results.

Step 6: Stop.

All the heuristics and experiments were coded in MATLAB R2015b, and were executed on an Intel core i7 processor with a $3.40 \mathrm{GHz}$ CPU and 8 GB RAM.

\section{Computational experiments and results}

In this Section, we conduct rigorous computational experiments to illustrate our findings and evaluate the performance of the proposed heuristics methods in comparison to the GRG solution. The problem setting that is designed for the numerical study conducted under ideal 
and recovery conditions is described in Section 7.1. To present further insights, a significant number of randomly generated test problems are experimented. In Section 7.2, we compare our results with the solutions obtained from the GRG method. A scenario-based analysis is presented in Section 7.3. We further conduct a sensitivity analysis in Section 7.4 where the impact of the delivery delay and partial quantity loss on the model costs are investigated through separate random experimentations. Finally, a detailed numerical analysis, with a special focus on the cases where number of cycles in the recovery time window is unknown, is performed in Section 7.5.

\subsection{Numerical examples for the ideal and recovery plans}

We first focus on the ideal plan problem i.e. no disruption in the system. Although the values for the key parameters in our experiments are randomly generated, to prevent production of non-meaningful results, it is guaranteed that the selected parameter values and ranges do not cause too trivial and non-practical cases (Paul et al. 2014c, 2015b, 2016b).

$D=100,000, P_{s}=240,000, P_{m}=120,000, N=2, H_{1}=0.5, H_{2}=0.8, S_{1}=80, S_{2}=120, S_{t s}=$ 0.0008 , and $S_{t m}=0.0006$.

Table 1: Sample instance for each disruption situation

\begin{tabular}{|c|l|c|}
\hline Instance number & \multicolumn{1}{|c|}{ Consequence } & Parameter \\
\hline 1 & Only delivery delay & $T_{d}=0.05$ \\
\hline 2 & Only partial quantity loss & $f=0.60$ \\
\hline 3 & Both delivery delay and partial quantity loss & $\begin{array}{c}T_{d}=0.04 \\
f=0.30\end{array}$ \\
\hline
\end{tabular}

Using the above parameter values and from Eqs. (6) and (7), the EPQ for both the manufacturer and supplier is determined. Subsequently, the total annual holding and set-up costs of the system is determined (here, \$7,746). Finally, an ideal time between two consecutive lots for the supplier and manufacturer is found by using Eqs. (8) and (9), respectively. Our results for the ideal plan are listed as follows.

$Q^{*}=10,328$ units; $q^{*}=5,164$ units; $T C=\$ 7,746 ; T_{s}=0.0078$ year; $T_{m}=0.0080$ year. 
Table 2: Recovery plan for the sample instances

\begin{tabular}{|c|c|c|c|c|c|}
\hline $\begin{array}{l}\text { Instance } \\
\text { number }\end{array}$ & \multicolumn{2}{|c|}{ Recovery plan } & $\begin{array}{c}\text { Total } \\
\text { backorders }\end{array}$ & $\begin{array}{l}\text { Total lost } \\
\text { sales cost }\end{array}$ & Total cost \\
\hline 1 & $\begin{array}{l}X_{1}=10,328 \\
X_{2}=7,696 \\
X_{3}=10,328 \\
X_{4}=10,328 \\
X_{5}=10,328\end{array}$ & $\begin{array}{l}Y_{1}=5,164 \\
Y_{2}=3,848 \\
Y_{3}=5,164 \\
Y_{4}=5,164 \\
Y_{5}=5,164\end{array}$ & $10,995.7$ & $13,160.2$ & $26,066.9$ \\
\hline 2 & $\begin{array}{l}X_{1}=10,328 \\
X_{2}=11,878 \\
X_{3}=11,878 \\
X_{4}=11,878 \\
X_{5}=11,878\end{array}$ & $\begin{array}{l}Y_{1}=2,066 \\
Y_{2}=5,939 \\
Y_{3}=5,939 \\
Y_{4}=5,939 \\
Y_{5}=5,939\end{array}$ & $1,105.8$ & 0 & $3,289.1$ \\
\hline 3 & $\begin{array}{l}X_{1}=10,328 \\
X_{2}=10,270 \\
X_{3}=10,270 \\
X_{4}=10,270 \\
X_{5}=10,270\end{array}$ & $\begin{array}{l}Y_{1}=3,615 \\
Y_{2}=5,135 \\
Y_{3}=5,135 \\
Y_{4}=5,135 \\
Y_{5}=5,135\end{array}$ & $4,611.7$ & $16,652.2$ & $23,209.6$ \\
\hline
\end{tabular}

After characterizing the ideal plan, we turn our attention to the decision-making problem under the recovery plan, where a sudden disruption affects the transportation system. The values of the input parameters are set as $B=20, L=10$, and $n=5$ according to the initial tests. To obtain the insights, a significant number of random disruption instance problems is used and a computational experiment is performed. For a sample result presentation, we focus on an exemplary instance for each disruption consequence (see Table 1).

The results for three sample instances are shown in Table 2, which shows the recovery plan, total backorders, lost sales cost, and total supply chain cost during the recovery time window. Both backorders and lost sales appear in instances 1 and 3, while only the backorders cost is presented in instance 2. Following the properties developed in Section 4.3 , this can be explained by the fact that although the optimal recovery plan incorporates both backorders and lost sales, it is capable to revise the plan by using only backorders. The 
reason and condition for incorporating the backorders and lost sales will be investigated in Sections 7.3 and 7.4 through a scenario-based analysis and sensitivity analysis.

\subsection{Results comparison}

To evaluate the quality of the solutions of three presented heuristics, the GRG non-linear method is used as a benchmark. GRG is a standard solution technique for solving constrained non-linear mathematical programming problems (Gabriele and Ragsdell 1977). To design our experiments, the following options in the GRG method is considered:

Convergence: 0.0001, Derivatives: forward, Population size: 100, Random seed: 0, Constraint precision: 0.000001 .

Then, the results obtained from both the heuristics and GRG method are compared for a reasonable number of random test problems. To this end, we determine the average percentage of deviation of the results by using Eq. (23), which is commonly used in the literature (Paul et al. 2014c, 2015b, 2016b).

Average percentage of deviation:

$=\frac{1}{M} \sum\left[\frac{\mid \text { Total profit from heuristc-Total profit from GRG } \mid}{\text { Total profit from GRG }} \times 100 \%\right]$.

Here, $M$ denotes the number of test problems.

The results obtained from our heuristics were compared against the GRG non-linear method using Excel solver for 100 random disruption test problems. The test problems are generated from a random uniform distribution by varying the disruption data. In this comparison experiment, the average percentage of deviation, which was calculated through Eq. (23), for the results obtained from the two approaches, is $0.0017 \%$. This deviation is negligible, due probably to the rounding of the values of the decision variables. On the consistency of the solutions, the results are analysed by changing the data on delivery delay and fraction of quantity loss, as shown in Figures 3 and 4, respectively. For all ranges of data, our heuristic approaches produce consistent results compared to the GRG method. In addition, the solution time of both approaches is negligible albeit our proposed approach is easier to implement. This shows the effectiveness of the proposed solution. 


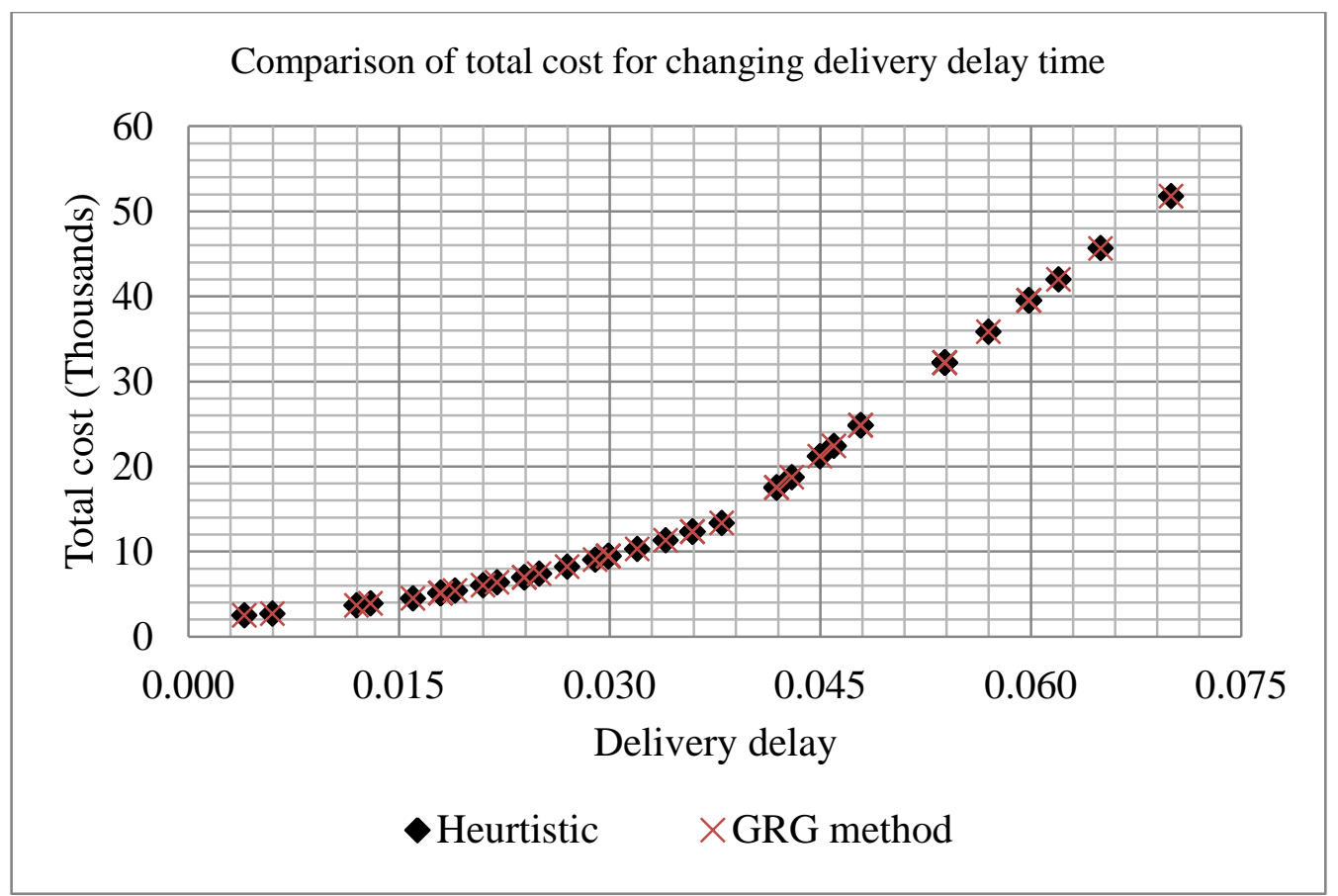

Fig. 3: Comparison of total cost with changing delivery delay time

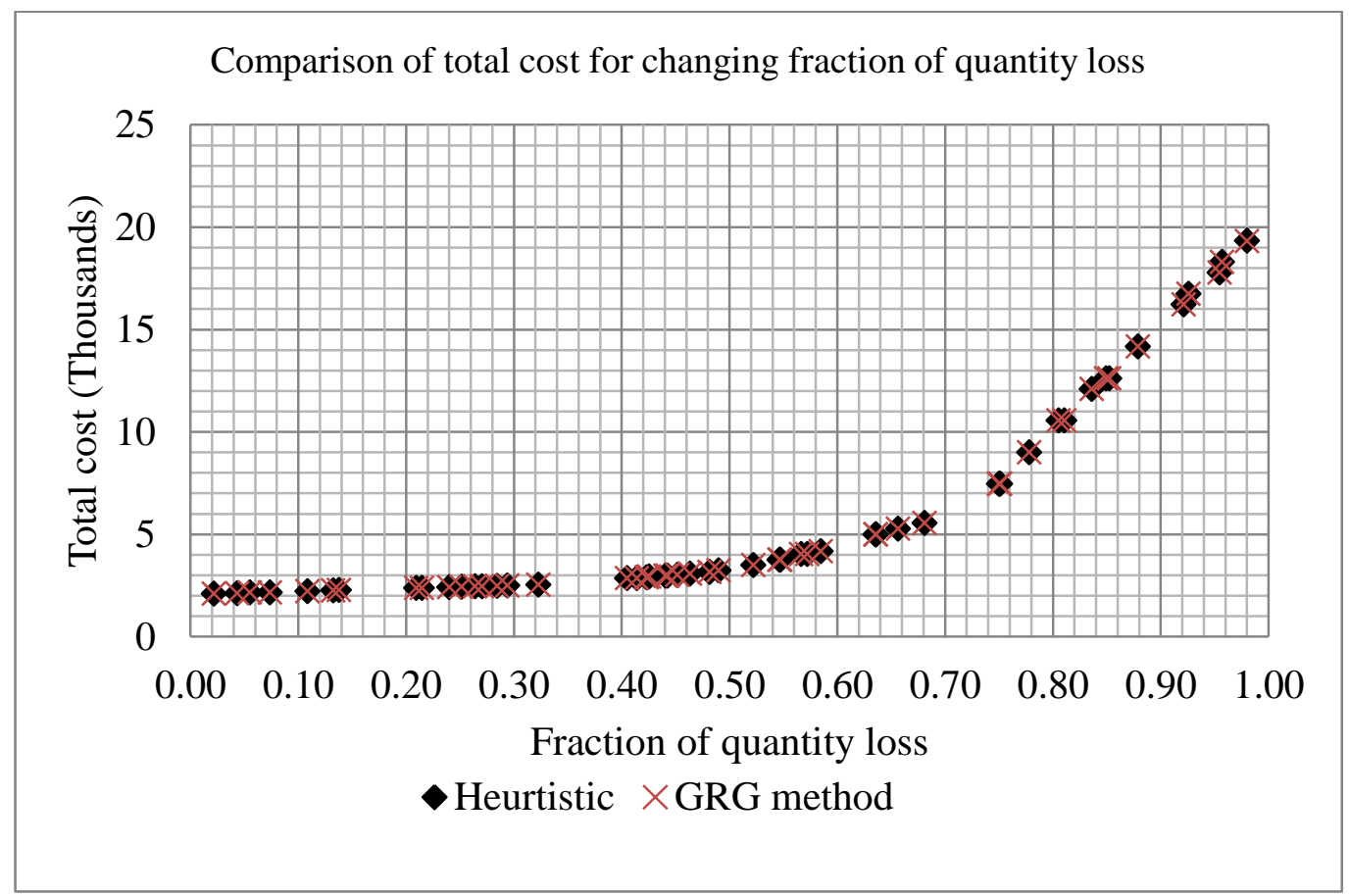

Fig. 4: Comparison of total cost with changing fraction of quantity loss

\subsection{Scenario-based analysis}

After comparing and validating our proposed heuristics, the results for the different types of disruption scenarios are analysed. To this end, we randomly generate the scenarios from all 
possible consequences arising from sudden transportation disruptions and categorise them as low, medium, and high profile, as shown in Table 3.

50 random test problems are generated by using a uniform probability distribution for each category and consequence to analyze the results. The values of the other parameters are hold constant as presented in Section 7.1.

Table 3: Scenarios of transportation disruption

\begin{tabular}{|l|c|c|c|}
\hline \multirow{2}{*}{ Category } & \multicolumn{3}{|c|}{ Consequence } \\
\cline { 2 - 4 } & Delivery delay & Quantity loss & Delivery delay and quantity loss \\
\hline Low & $T_{d}=[0.0001,0.03]$ & $f=[0.0001,0.4]$ & $T_{d}=[0.0001,0.03] ; f=[0.0001,0.4]$ \\
\hline Medium & $T_{d}=[0.03,0.06]$ & $f=[0.4,0.8]$ & $T_{d}=[0.03,0.06] ; f=[0.4,0.8]$ \\
\hline High & $T_{d}=[0.06,0.08]$ & $f=[0.8,1]$ & $T_{d}=[0.06,0.08] ; f=[0.8,1]$ \\
\hline
\end{tabular}

Figures 5 to 8 summarize the results, which show the average total cost, the backorder and lost sales costs for each category of disruption scenario. When the consequence is only a delivery delay, as shown in Figure 5, it is observed that the total lost sales cost is significantly higher in a high delay scenario and the average backorder cost flattens for the medium to high scenarios. This is because the lost sales cost becomes more attractive than the backorder cost.

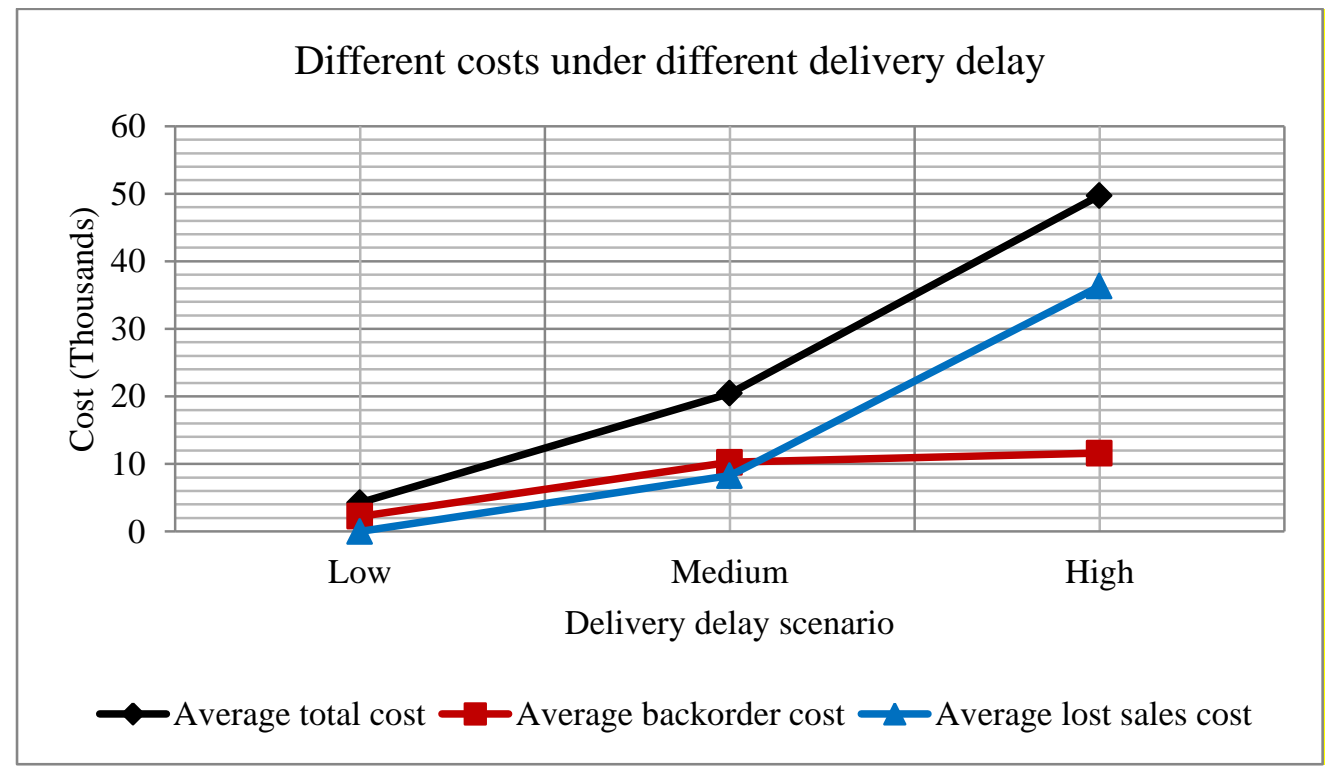

Fig. 5: Costs for different delivery delay scenarios 


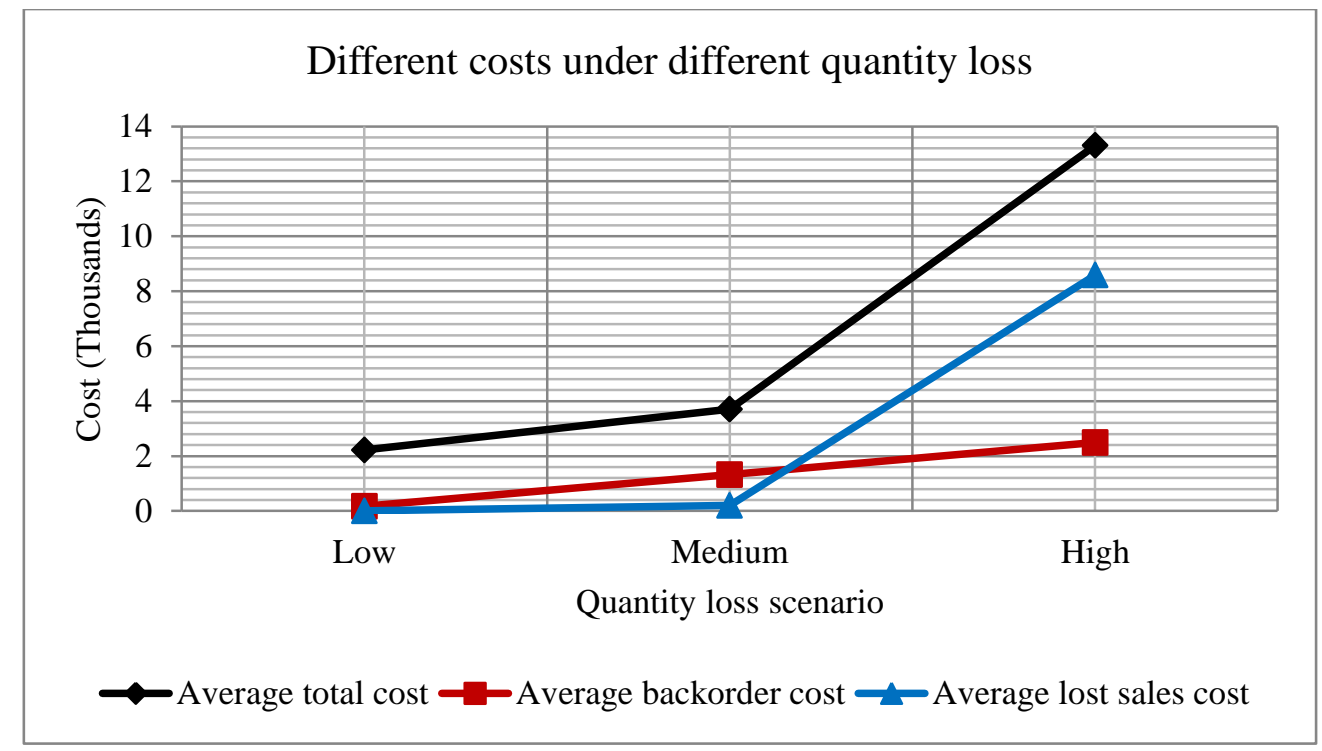

Fig. 6: Costs for different quantity loss scenarios

When the disruption consequence is only a quantity loss, as shown in Figure 6, the lost sales cost increases slowly from the low to high profile scenario and after that the increment rate becomes higher. This is because the lost sales become more attractive with higher quantity loss and in the low and medium quantity loss scenarios, the backorders are more attractive and are incorporated in the revised plan.

For both delivery delay and quantity loss, as shown in Figure 7, the lost sales are mostly incorporated in the revised plan. This is because the revised plan is not capable of dealing with only backorders and lost sales becomes more attractive.

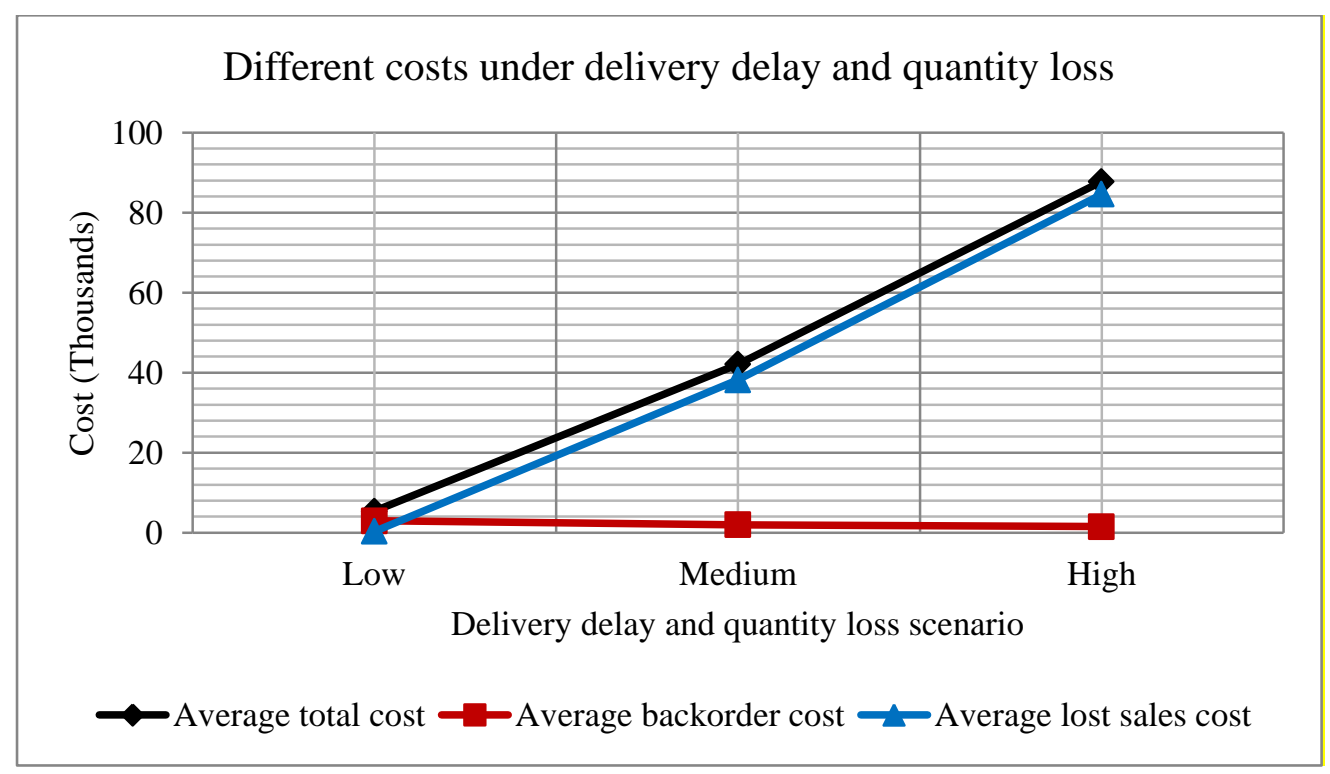

Fig. 7: Cost for both delivery delay and quantity loss scenario 


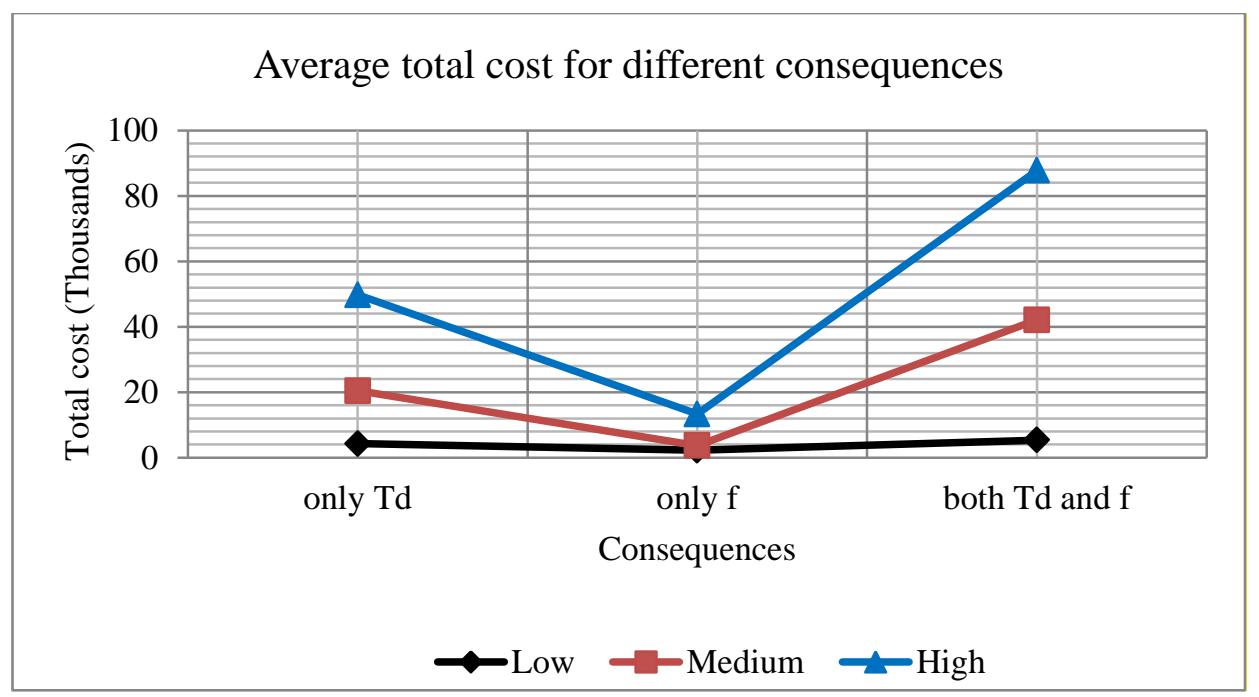

Fig. 8: Average total cost for different disruption consequences

To measure the severity of disruption, it is observed that the total cost is significantly higher when consequences are both delivery delay and quantity loss, as shown in Figure 8. Between the delivery delay and quantity loss, the total cost is higher when the consequence is delivery delay. This is because of the systems having only some idle time between two consecutive lots, which makes it sensitive to dealing with delivery delay, and lost sales are incorporated when the delivery delay is much smaller.

\subsection{Random experimentation and sensitivity analysis}

After evaluating the performance of the proposed heuristics, a random experimentation is conducted and sensitivity analysis is performed to characterize the impact of the parameters on the final solutions. To this aim, we conduct a random experimentation for 1,000 random test problems that are generated for each disruption scenario from a random uniform distribution. In what follows, the results of random experimentation are summarised.

It is observed that the both the delivery delay and fraction of quantity loss have significant impact on the model costs. Figure 9 presents the impact of delivery delay on backorders, lost sales, and total cost. We also observe that after a disruption, the system is capable of revising the production plan only by using the backorders until the delay is 0.039 . The recovery plan incorporates both the lost sales and backorders options. Due to incorporating only the backorders in the revised plan, the total cost increases slowly when the delivery delay is less than 0.039 . When the delivery delay exceeds 0.039 , the total cost increases at a higher rate with delivery delay because the lost sales cost has also been 
incorporated, along with the backorders, in the revised plan. This explanation supports Property 3.

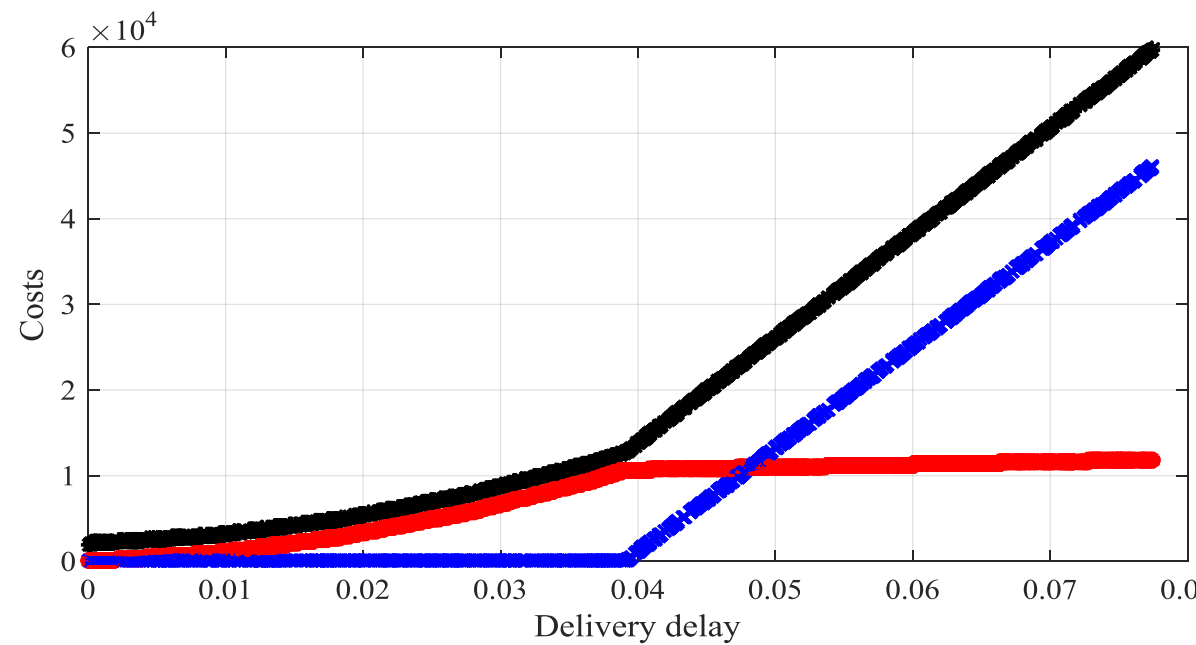

$\longrightarrow$ Total cost $\longrightarrow$ Total backorder cost $\longrightarrow$ Total lost sales cost

Fig. 9: Change in cost from delivery delay

We run 1,000 random test problems for delivery delay due to a transportation disruption using a uniform distribution in the range [0.0001, 0.08]. Figure 10 shows the total cost pattern for the test problems. The mean and standard deviation of the total cost are 21,046 and 18,299, while the maximum and minimum total cost values are 59,768 and 2,012, respectively.

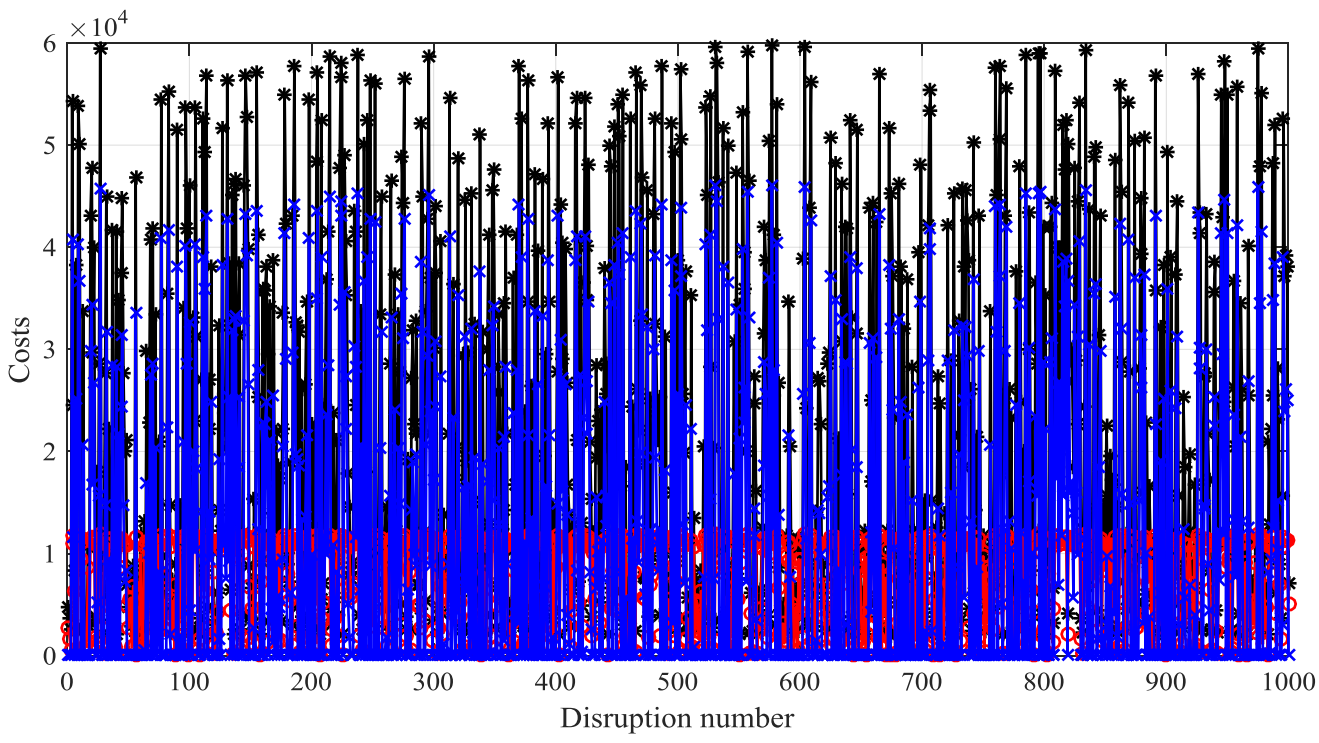

* Total cost $\quad$ - Total backorder cost $\quad \nVdash$ Total lost sales cost

Fig. 10: Random experimentation for only delivery delay 
Figure 11 presents the impact of the fraction of quantity loss on backorders, lost sales, and total cost. It can be seen that the system is capable to revise the plan by incorporating only backorders until the fraction of quantity loss is 0.72 . When the fraction exceeds 0.72 , the recovery plan incorporates both the lost sales and backorders options. In addition, due to incorporating only the backorders in the revised plan, the total cost increases slowly when the fraction of quantity loss is less than 0.72 . It is because the lost sales cost has no impact on the recovery plan and only backorders are incorporated in the revised plan. In contrast, when the fraction of quantity loss exceeds 0.72 , the total cost increases at a higher rate with the fraction of quantity loss because the lost sales cost has also been incorporated in the recovery plan. This explanation supports Property 4.

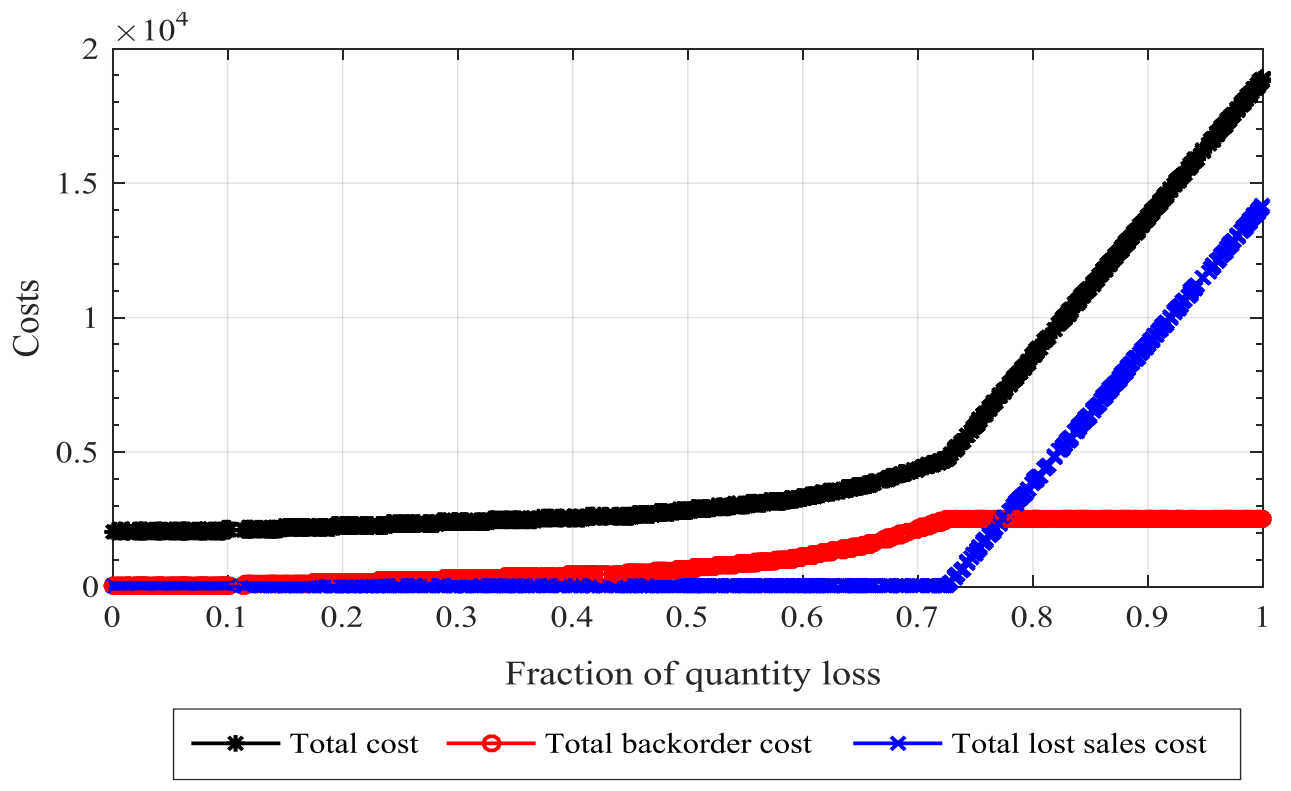

Fig. 11: Change in cost with fraction of quantity loss

Similar to the previous experiments, 1,000 random test problems are generated for the fraction of quantity loss due to a transportation disruption using a uniform distribution in the range $[0.0001,1]$. The total cost pattern for all test problems is presented in Figure 12. The mean and standard deviation of the total cost are 4,901 and 4,358, while the maximum and minimum total cost values are 18,862 and 2,000 respectively.

Finally, to examine the third scenario under transportation disruption where both the delivery delay and fraction of quantity loss exist, 1,000 random test problems are generated using a uniform distribution in the range $[0.0001,0.08]$ and $[0.0001,1]$ for delivery delay and fraction of quantity loss, respectively. The variation in the total cost in the presence of both a delivery delay and a fraction of quantity loss are presented in Figure 13. In this scenario, it is 
observed that the impact of both consequences on the total cost is higher than the earlier two scenarios, where a single consequence is presented.

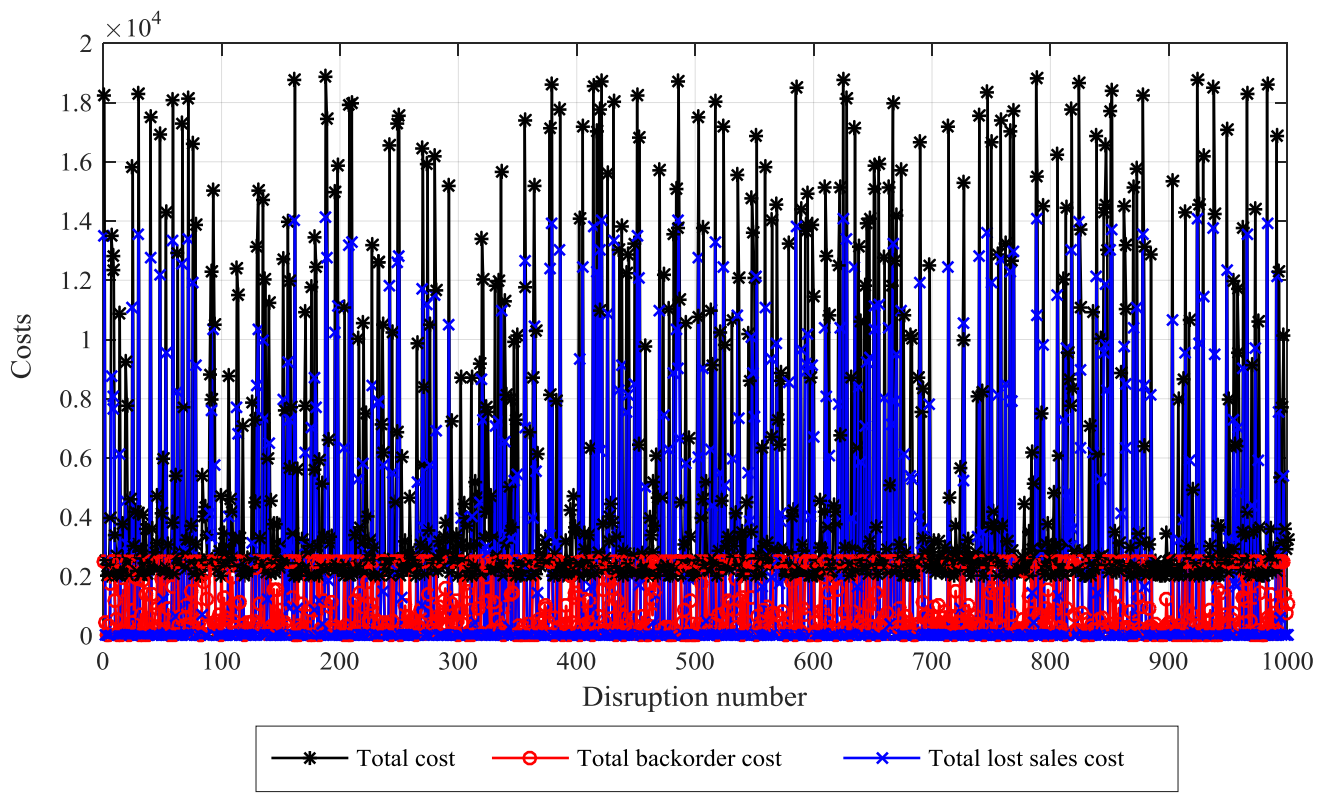

Fig. 12: Random experimentation for fraction of quantity loss

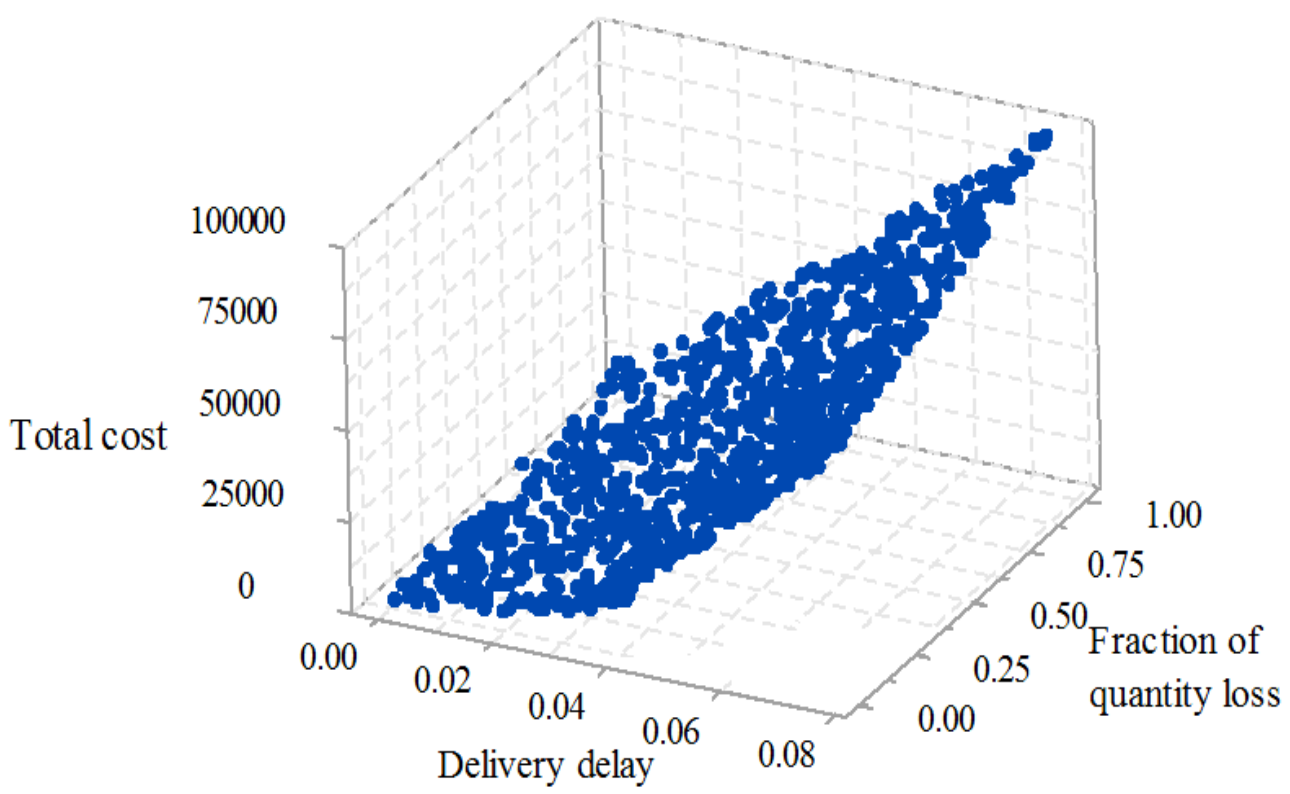

Fig. 13: Change in total cost from both delivery delay and fraction of quantity loss 


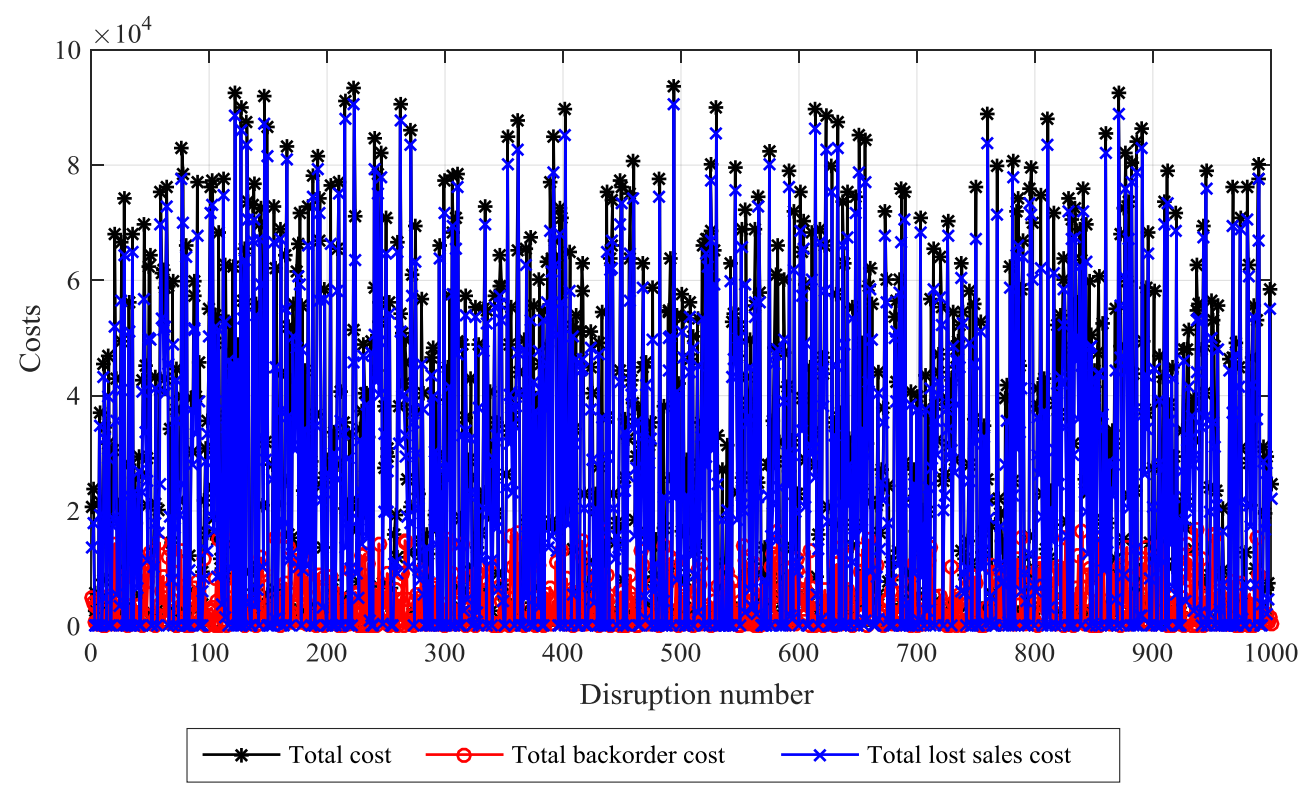

Fig. 14: Random experimentation for both delivery delay and fraction of quantity loss

The total cost pattern for all the test problems is presented in Figure 14. The total cost for this situation is much greater than the other two situations because of the presence of both delay and quantity losses in the revised plan. The mean and standard deviation of the total cost are now 32,660 and 26,031, while the maximum and minimum total cost values are 93,633 and 2,080, respectively.

\subsection{Analysing results for unknown number of cycles in the recovery window}

In this Section, the result for determining maximum number of recovery cycles is analysed and the impact of different parameters is investigated. We used the same input data presented in Section 7.1 and followed the procedure which is developed in Section 5.2 to analyse the key relevant results.

Figures 15 and 16 present the changes of $n_{-} \max$ with delivery delay and partial quantity loss respectively. As expected, it is observed that the number of recovery cycle increases with increasing both delivery delay and quantity loss. Our results suggest that there is a positive dependency among the magnititude of disruptions and the requiered number of recovery cycle considered in recoverly planning, enabling the operations managers to effectively mitigate the negative impacts of unpredictable transportation disruptions. 


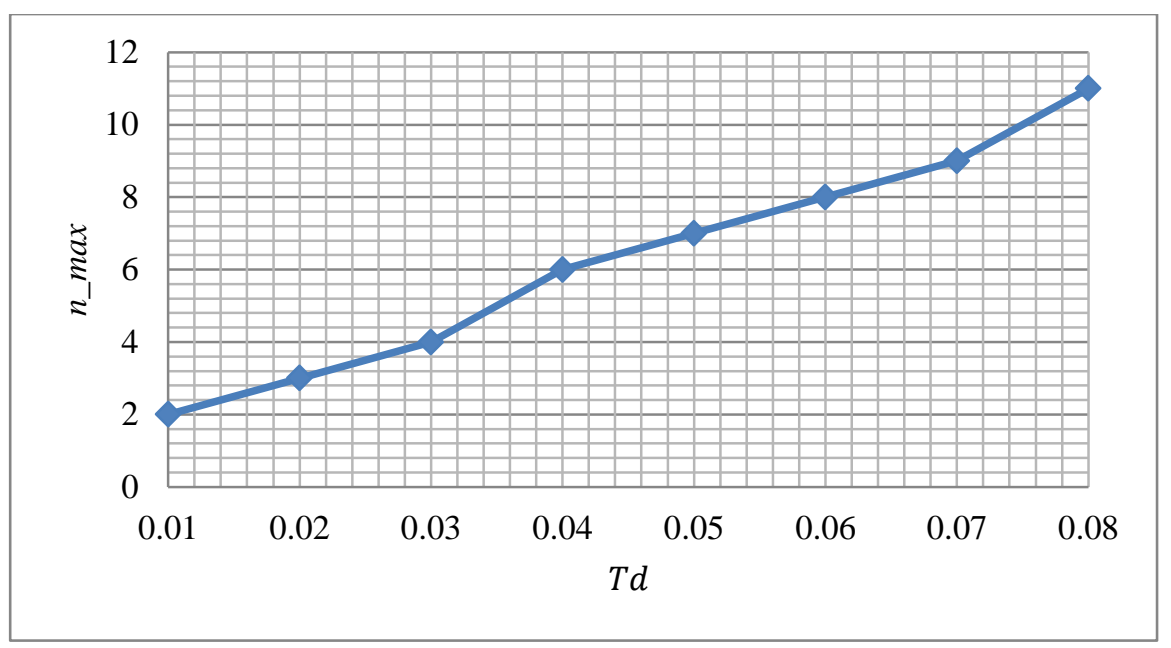

Fig. 15: Delivery delay vs $n \_\max$

Further analysis is performed to study how total backorder and lost sales costs are influenced by the number of recovery cycles. Figure 17 and 18 summarise the important results of this study and numerically reaffirm the concavity of total backorder and lost sales cost functions studied in present research. This highlights the need for determining an optimal number of recovery cycles based on the real-time information on partial quantity loss $(f)$ and delivery delay $\left(T_{d}\right)$ which are perceived only after occurrence of a transportation disruption.

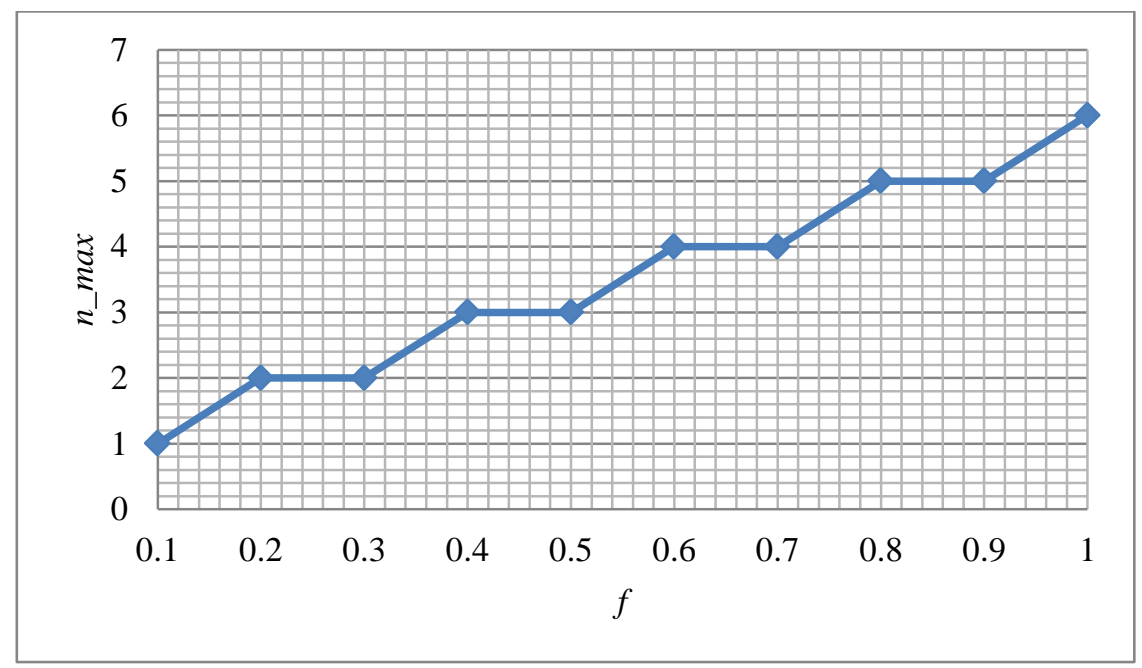

Fig. 16: Partial quantity vs lossn_max

As a summary, Figure 17 demonstrates the changes of total backorder and lost sales cost with $n$ for different values of $T_{d}$. For different values of $T_{d}$, different number of recovery cycles are identified. For example, when $T_{d}$ is 0.01 , the optimal number of recovery cycle is 2 , then with increasing the value of $T_{d}$, the number of recovery cycles increases. For $T_{d}=$ 0.07 , the number of recovery cycle increases to 9 . 


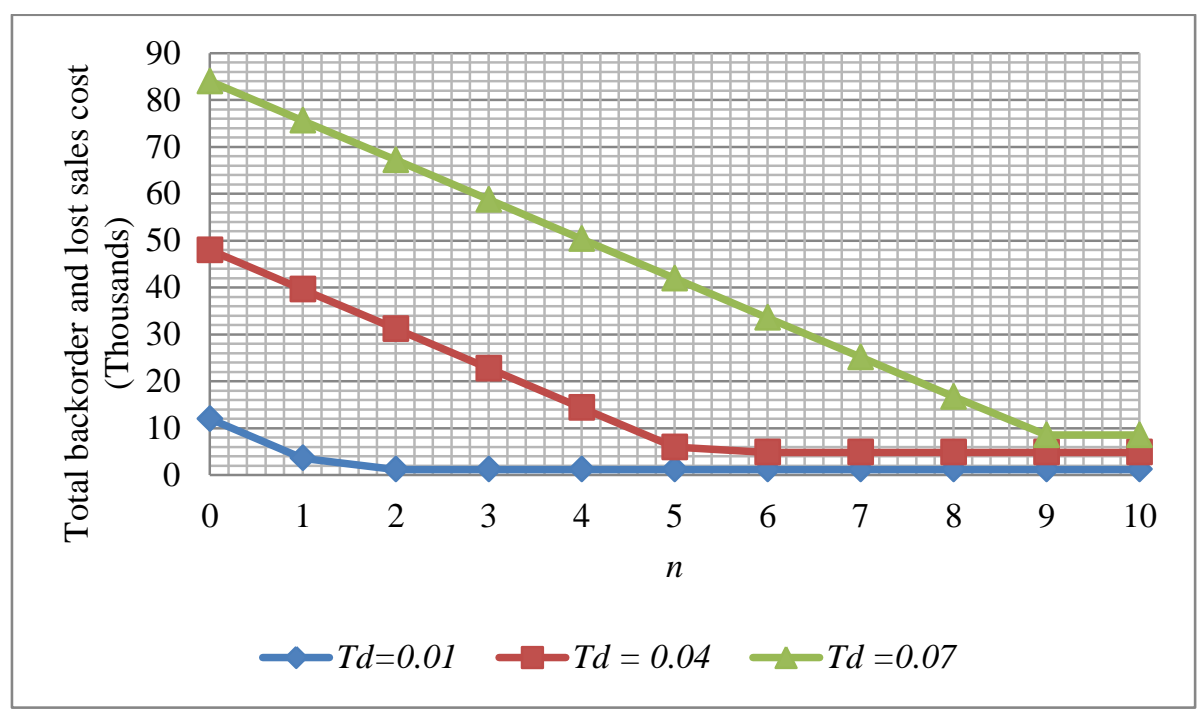

Fig. 17: Total backorder and lost sales cost vs $n$ for different $T_{d}$

Figure 18 shows the changes of total backorder and lost sales cost with $n$ for different values of $f$. Like the delivery delay $\left(T_{d}\right)$ case analysis, our observation suggests that the optimal number of recovery cycles varies for different values of $f$, respectively. For example, when $f$ is 0.3 , the optimal number of recovery cycle is 3 , then with increasing the value of $f$, the number of recovery cycles increases. For $f=1$, the number of recovery cycle increases to 7.

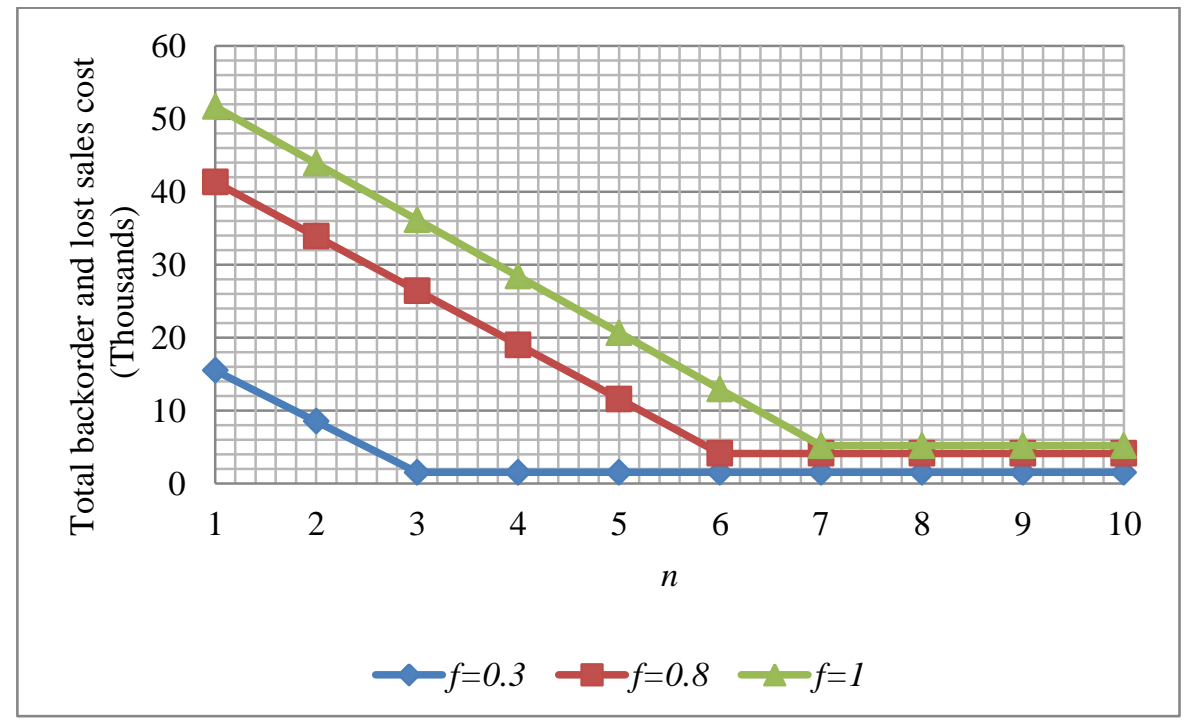

Fig. 18: Total backorder and lost sales cost vs $n$ for different $f$ 


\section{Conclusion}

The main purpose of this study was to develop a recovery model for managing transportation disruption in a lean, two-stage supplier-manufacturer supply chain, with no inventory buffer. The model considered both delivery delay and quantity losses as the consequences of a sudden transportation disruption, to make the problem more general and applicable in reallife. Due to the costly commercial optimization software, and complexity and computational intensity of the optimal solution, three heuristics were developed to solve the model for all possible disruption scenarios. After formulating the problem and characterizing the main properties of the models, random experimentation was conducted to analyse the results on the randomly generated test problems. To ensure the effectiveness of the proposed recovery policies and enhance the practicality of the developed model, the fixed number of recovery cycles assumption has been relaxed and suitable heuristic procedures for determining the best number of recovery cycles in the event of transportation disruption have been presented. The heuristics results were compared with the GRG method. It was observed that the average percentage deviation of the results was negligible at $0.0017 \%$. We also performed a scenariobased analysis, which explored insights into the model properties. The heuristics can produce consistent results for all ranges of data on delivery delay and the fraction of quantity loss.

Compared to the previous studies in the literature, our paper is one of the first efforts to investigate recovery planning in a two-stage supplier-manufacturer supply chain under the presence of both delivery time delay and fractional quantity loss resulted by sudden transportation disruptions. However, several aspects could be introduced into our approach, as future work, to make the problem more realistic and strengthen the quality of our results.

First, as the intent of this paper was to particularly look at a lean supply chain, safety stock has invariably been removed from consideration in the mathematical model. However, considering the safety-stock level in a general supply chain setting, analysing the effect of a transportation disruption on safety stock, and finding the optimum safety-stock level to minimize the disruption effects would be a challenging but potential future research direction. In the current model, for simplicity in the model building and ease of understanding in the model implementation, the fraction of quantity loss is considered as a constant. However, in reality, it varies with time due to the on-going effects of perishability. So, another interesting future work could be incorporating a variable fraction for the quantity loss which can depend on time, stock level, and the intensity of disruptions. Next, investigating the lead-time factors and analysing the effect of a transportation disruption on lead-time and recovery plan is another future research step. Finally, supply chains, in practice, comprise multiple tiers. 
Hence, extending our problem setting to a multi-tier supply chain problem with multiple suppliers, manufacturers, and others, and multiple products can be another interesting research avenue.

\section{References}

Alcantara, P., \& Riglietti, G. (2015). Supply Chain Resilience Report. The Business Continuity Institute, pp. 1-36.

Ansaripoor, A. H., Asian, S., Faghih-Roohi, S., Lee, P. T. W., \& Hu, Z. H., (2017). Risk quantification in maritime fleet planning problem: A CVaR approach. Working Paper.

Asian, S., \& Nie, X. (2014). Coordination in supply chains with uncertain demand and disruption risks : existence, analysis, and insights. IEEE Transactions on Systems, Man, and Cybernetics: Systems, 44(9), 1139-1154.

Asian, S., Ertek, G., Haksoz, C., Pakter, S., \& Ulun, S. (2016). Wind turbine accidents: A data mining study. IEEE Systems Journal. doi:10.1109/JSYST.2016.2565818

Asian, S., Hemati, M., \& Samandizadeh, K. (2009). Strategic planning evaluation in manufacturing companies through fuzzy analytic hierarchy process (FAHP). Journal of Industrial Management, 4(7), 1-20.

Baghalian, A., Rezapour, S., \& Farahani, R. Z. (2013). Robust supply chain network design with service level against disruptions and demand uncertainties: a real-life case. European Journal of Operational Research, 227, 199-215.

Banerjee, A. (1986). A joint economic-lot-size model for purchaser and vendor. Decision Sciences, 17(3), 292-311.

Blome, C., \& Schoenherr, T. (2011). Supply chain risk management in financial crises-a multiple case-study approach. International Journal of Production Economics, 134(1), $43-57$.

Chen, J., Sohal, A. S., \& Prajogo, D. I. (2013). Supply chain operational risk mitigation: a collaborative approach. International Journal of Production Research, 51(7), 21862199.

Chen, L.-M., Liu, Y. E., \& Yang, S.-J. S. (2015). Robust supply chain strategies for recovering from unanticipated disasters. Transportation Research Part E: Logistics and Transportation Review, 77, 198-214.

Chen, J.M. and Chen, T.H., 2005. The multi-item replenishment problem in a two-echelon supply chain: the effect of centralization versus decentralization. Computers \& Operations Research, 32(12), 3191-3207. 
Choi, T. M., Wallace, S. W., \& Wang, Y. (2016). Risk management and coordination in service supply chains: information, logistics and outsourcing. Journal of the Operational Research Society, 67(2), 159-164.

Choi, T. M., Chiu, C. H., \& Chan, H. K. (2016). Risk management of logistics systems. Transportation Research Part E: Logistics and Transportation Review, 90, 1-6, http://dx.doi.org/10.1016/j.tre.2016.03.007.

Chopra, S., \& Sodhi, M. S. (2014). Reducing the risk of supply chain disruptions. MIT Sloan Management Review, 55(3), 73-80.

Chowdhury, P., Lau, K. H., \& Pittayachawan, S. (2016). Supply risk mitigation of small and medium enterprises: a social capital approach. In Proc. of 21 st International Symposium on Logistics (pp. 37-44). Centre for Concurrent Enterprise, Nottingham University.

Chung, S. H., Tse, Y. K., \& Choi, T. M. (2015). Managing disruption risk in express logistics via proactive planning. Industrial Management \& Data Systems, 115(8), 1481-1509.

Daskin, M.S., Snyder, L.V. and Berger, R.T., 2005. Facility location in supply chain design. Logistics Systems: Design and optimization, 39-65.

Do, N. A. D., Nielsen, I. E., Chen, G., \& Nielsen, P. (2016). A simulation-based genetic algorithm approach for reducing emissions from import container pick-up operation at container terminal. Annals of Operations Research, 242, 285-301.

Faghih-Roohi, S., Ong, Y. S., Asian, S., \& Zhang, A. N. (2016). Dynamic conditional valueat-risk model for routing and scheduling of hazardous material transportation networks. Annals of Operations Research, 247(2), 715-734.

Fahimnia, B., Tang, C. S., Davarzani, H., \& Sarkis, J. (2015). Quantitative models for managing supply chain risks: a review. European Journal of Operational Research, 247(1), 1-15.

Gabriele, G. A., \& Ragsdell, K. M. (1977). The generalized reduced gradient method : a reliable tool for optimal design. Journal of Engineering for Industry, 99(2), 394-400.

Giunipero, L. C., \& Eltantawy, R. A. (2004). Securing the upstream supply chain: a risk management approach. International Journal of Physical Distribution \& Logistics Management, 34(9), 698-713.

Guiffrida, A. L., \& Jaber, M. Y. (2008). Managerial and economic impacts of reducing delivery variance in the supply chain. Applied Mathematical Modelling, 32(10), 21492161.

Guo, S., Zhao, L., \& Xu, X. (2016). Impact of supply risks on procurement decisions. Annals of Operations Research, 241(1), 411-430. 
Hendricks, K. B., \& Singhal, V. R. (2003). The effect of supply chain glitches on shareholder wealth. Journal of Operations Management, 21(5), 501-522.

Hishamuddin, H. (2013). Optimal Inventory Policies for Multi-Echelon Supply Chain Systems with Disruption. The University of New South Wales, Canberra, Australia.

Hishamuddin, H., Sarker, R., \& Essam, D. (2012). A disruption recovery model for a single stage production-inventory system. European Journal of Operational Research, 222(3), $464-473$.

Hishamuddin, H., Sarker, R., \& Essam, D. (2013). A recovery model for a two-echelon serial supply chain with consideration of transportation disruption. Computers \& Industrial Engineering, 64(2), 552-561.

Hishamuddin, H., Sarker, R., \& Essam, D. (2014). A recovery mechanism for a two echelon supply chain system under supply disruption. Economic Modelling, 38, 555-563.

Ho, W., Zheng, T., Yildiz, H., \& Talluri, S. (2015). Supply chain risk management: a literature review. International Journal of Production Research, 53(16), 5031-5069.

Hu, F., Lim, C. C., Lu, Z., \& Sun, X. (2013). Coordination in a single-retailer two-supplier supply chain under random demand and random supply with disruption. Discrete Dynamics in Nature and Society, (Article ID 484062), 1-12.

Kim, S. W. (2013). A supply chain contract with flexibility as a risk-sharing mechanism for demand forecasting. International Journal of Systems Science, 44, 1134-1149.

Kim, Y., Chen, Y. S., \& Linderman, K. (2015). Supply network disruption and resilience: a network structural perspective. Journal of Operations Management, 33, 43-59.

Khazaei Pool, J., Arabzad, S., Asian, S., Fahimi, M., \& Verij Kazemi, R. (2017a). Employing fuzzy ANP for ranking the personality of international brands in the sports shoe industry. Journal of Modelling in Management. doi:10.1108/JM2-02-2016-0009

Khazaei Pool, J., Asian, S., Arabzad, S. M., Balouei Jamkhaneh, H., \& Kia Lashaki, J. (2017b). Development of a model to analyze the factors affecting RFID technology acceptance in small and medium-sized enterprises. International Journal of Services and Operations Management, (in press).

Li, J., Wang, S., \& Cheng, T. C. E. (2010). Competition and cooperation in a single-retailer two-supplier supply chain with supply disruption. International Journal of Production Economics, 124(1), 137-150.

Li, N., Chen, G., Govindan, K., \& Jin, Z. (2015). Disruption management for truck appointment system at a container terminal: a green initiative. Transportation Research Part D: Transport and Environment, (in press), 1-13. doi:10.1016/j.trd.2015.12.014 
Lu, D., Ding, Y., Asian, S. and Paul, S.K., 2017. From Supply Chain Integration to Operational Performance: The Moderating Effect of Market Uncertainty. Global Journal of Flexible Systems Management, pp.1-18.doi:10.1007/s10845-016-1200-7.

Paul, S. K., Sarker, R., \& Essam, D. (2017). A quantitative model for disruption mitigation in a supply chain. European Journal of Operational Research, 257(3), 881-895.

Rezaei Somarin, A., Asian, S., \& Chen, S. (2016). Dynamic priority repair policy for service parts supply chain. In 2016 IEEE international conference on industrial engineering and engineering management (IEEM) (pp. 798-802). IEEE

Rezaei Somarin, A., Chen, S., Asian, S., \& Wang, D. Z. (2017a). A heuristic stock allocation rule for repairable service parts. International Journal of Production Economics, 184, 131-140.

Rezaei Somarin, A., Asian, S., Jolai, F. and Chen, S., 2017. Flexibility in service parts supply chain: a study on emergency resupply in aviation MRO. International Journal of Production Research, pp.1-16. doi:10.1080/00207543.2017.1351640

Ray, P., \& Jenamani, M. (2016). Sourcing decision under disruption risk with supply and demand uncertainty: a newsvendor approach. Annals of Operations Research, 137(1), 237-262.

Sarker, R. A., \& Khan, L. R. (1999). An optimal batch size for a production system operating under periodic delivery policy. Computers \& Industrial Engineering, 37(4), 711-730.

Shao, X. F., \& Dong, M. (2012). Supply disruption and reactive strategies in an assemble-toorder supply chain with time-sensitive demand. IEEE Transactions on Engineering Management, 59(2), 201-212.

Sharifkhani, M., Khazaei Pool, J., \& Asian, S. (2016). The impact of leader-member exchange on knowledge sharing and performance: An empirical investigation in the oil and gas industry. Journal of Science and Technology Policy Management, 7(3), 289305.

Snyder, L. V, Atan, Z., Peng, P., Rong, Y., Schmitt, A. J., \& Sinsoysal, B. (2016). OR/MS models for supply chain disruptions: a review. IIE Transactions, 48(2), 89-109.

Tang, O., Musa, S. N., \& Li, J. (2012). Dynamic pricing in the newsvendor problem with yield risks. International Journal of Production Economics, 139, 127-134.

Tavakoli, M. R., Asian, S., \& Hemati, M. (2012). Using a fuzzy MADM in the Balanced SCORE card for the performance improvement in Iranian steel industries. Iranian Journal of Trade Studies (IJTS), 16(61), 51-80.

Wagner, S. M., \& Silveira-Camargos, V. (2012). Managing risks in just-in-sequence supply 
networks: Exploratory evidence from automakers. IEEE Transactions on Engineering Management, 59(1), 52-64.

Wagner, S. M., \& Bode, C. (2008). An empirical examination of supply chain performance along several dimensions of risk. Journal of Business Logistics, 29(1), 307-325.

Weiss, H. J., \& Rosenthal, E. C. (1992). Optimal ordering policies when anticipating a disruption in supply or demand. European Journal of Operational Research, 59(3), 370382.

Wieland, A., \& Wallenburg, C. M. (2013). The influence of relational competencies on supply chain resilience: a relational view. International Journal of Physical Distribution \& Logistics Management, 43(4), 300-320.

Wilson, M. C. (2007). The impact of transportation disruptions on supply chain performance. Transportation Research Part E: Logistics and Transportation Review, 43(4), 295-320.

Xia, Y., Yang, M. H., Golany, B., Gilbert, S. M., \& Yu, G. (2004). Real-time disruption management in a two-stage production and inventory system. IIE Transactions, 36(2), $111-125$.

Yan, X., Zhang, M., Liu, K., \& Wang, Y. (2014). Optimal ordering policies and sourcing strategies with supply disruption. Journal of Industrial and Management Optimization, 10(4), 1147-1168.

Yang, Z., Aydın, G., Babich, V., \& Beil, D. R. (2009). Supply disruptions, asymmetric information, and a backup production option. Management Science, 55(2), 192-209.

Zhang, D., Sheng, Z., Du, J., \& Jin, S. (2013). A study of emergency management of supply chain under supply disruption. Neural Computing and Applications, 24(1), 13-20. 